\title{
Higher-Order Wavelet Reconstruction/Differentiation Filters and
}

\section{Gibbs Phenomena}

Richard Lombardini, ${ }^{\text {a,b }}$ Ramiro Acevedo, ${ }^{\text {a,c }}$ Alexander Kuczala, ${ }^{\text {a,d }}$ Kerry P. Keys, ${ }^{\text {a,e }}$ Carl P. Goodrich $^{\mathrm{a}, \mathrm{f}}$ and Bruce R. Johnson ${ }^{\mathrm{a}, \mathrm{g}}$

aDepartment of Chemistry, Smalley-Curl Institute and Laboratory for NanoPhotonics, Rice University, Houston, TX, USA 77005

bPresent Address: Department of Physics and Earth Sciences, St. Mary's University, San Antonio, TX, USA 78228

'Present Address: Physics Department, Wharton County Junior College, Sugar Land, TX, USA 77479

dPresent Address: Department of Physics, University of California San Diego, San Diego, CA, USA 92093

ePresent Address: Kenan-Flagler Business School, University of North Carolina, Chapel Hill, NC, USA 27599

fPresent Address: School of Engineering and Applied Sciences, Harvard University, Cambridge, MA, USA 02138

gCorresponding author, Email: johnson@rice.edu

\begin{abstract}
An orthogonal wavelet basis is characterized by its approximation order, which relates to the ability of the basis to represent general smooth functions on a given scale. It is known, though perhaps not widely known, that there are ways of exceeding the approximation order, i.e., achieving higher-order error in the discretized wavelet transform and its inverse. The focus here is on the development of a practical formulation to accomplish this first for 1D smooth functions, then for 1D functions with discontinuities and then for multidimensional (here 2D) functions with discontinuities. It is shown how to transcend both the wavelet approximation order and the 2D Gibbs phenomenon in representing electromagnetic fields at discontinuous dielectric interfaces that do not simply follow the wavelet-basis grid.
\end{abstract}


Keywords: wavelets, projection, reconstruction, Gibbs, multidimensional, boundary

\section{Introduction}

Wavelets are of general interest in developing systematically-improvable multiscale methods for solving differential equations in quantum mechanics, electromagnetism and many other applications. Orthogonal wavelet families such as those due to Daubechies [1] allow multiresolution description and compression of the solutions as well as fast forward and inverse wavelet transforms. These are efficient transformations between discretized real space functions and wavelet basis space coefficients. The forward (projection) transform is well controlled and may be carried out to a desired accuracy by using high-order numerical quadrature and, if needed, scale refinement procedures [2-4]. In contrast, the inverse (reconstruction) transform is usually described as being intrinsically limited by the approximation order of the wavelet family, which is a measure of its ability to approximate general smooth functions. Despite that common sentiment, Keinert and Kwon [5] and Neelov and Goedecker [6] demonstrated that one can beat this limit in reconstruction. Our group has recently generalized the latter results so that the reconstruction error can be tuned just as freely as the projection error [7]. There are particular consequences of this, as will be shown. For example, the increased tunability gives us the ability to use shorter-length wavelets while maintaining higher-order accuracy. More difficult generalizations are then pursued, e.g., maintaining high-order accuracy at function discontinuities, which requires overcoming the wavelet Gibbs phenomenon. This is then carried over to the $2 \mathrm{D}$ Gibbs phenomenon with an electromagnetic example possessing a dielectric interface that is curved rather than aligned with the wavelet basis grid. The strategy adopted is 
developed with an eye toward wavelet solution of differential equations in multiple dimensions with arbitrarily-shaped interfaces without being intrinsically restricted to low-order accuracy. The present work bears on how well such solutions can be represented in wavelet bases in the first place.

The first focus is on 1D function expansions in father wavelets or scaling functions, $\phi(x-$ $k), k$ an integer, and on inversion of a matrix $\mathbf{X}$ formed from projections of monomial powers ( $x-$ $\tau)^{p}$ onto this distributed basis. The parameter $\tau$ is a shift that may be varied so as to allow wavelet interpolation / extrapolation in reconstruction. The columns of $\mathbf{X}^{-1}$ can be applied to a series of neighboring coefficients to provide high-order real-space samples of the underlying function and its derivatives. The lengths of these local convolution filters and their error orders can be varied by varying the size of the original matrix. By translation invariance, the same filters can be applied to different groups of expansion coefficients of the same length. Furthermore, explicit formulae can be derived for the filters in terms of Lagrange interpolating polynomials and moments of $\phi(x)$.

A fundamentally different situation occurs when there are boundary conditions, e.g., potential energy discontinuities in quantum mechanics, dielectric function discontinuities in Maxwell's equations and so on. Wavelet basis functions generally have overlapping supports and are not particularly graceful in satisfying pointwise boundary conditions. For functions with discontinuities, one encounters the wavelet Gibbs phenomenon, where reconstructions using the regular basis functions show significant errors in the neighborhood of the discontinuity $[8,9]$. One potential remedy is to try to work only with basis functions, or parts of them, that fall on one 
side of the boundary at a time. Those few $\phi(x-k)$ with support straddling the boundary become truncated and lose their mutual orthogonality. It is known that linear combinations of them may be orthogonalized to produce special edge functions terminating at the boundary [10-12], but these "intervalized" bases are not fully compatible with high order reconstruction as pursued here.

Instead, the nonorthogonal tail functions are used directly. Their truncated moments may be calculated and used in a generalized version of the distributed-moment matrix $\mathbf{X}$, followed by its inversion to produce edge-adapted reconstruction filters. There are some caveats: (i) a number of different filters are required near the boundary, (ii) the inversion of the matrix is only done numerically and, consequently, (iii) greater care must be taken to avoid effects due to finite precision. Nevertheless, this straightforward extension successfully allows reconstruction and differentiation filters near the edge that exhibit the desired higher-order errors and avoid the undesired Gibbs phenomenon.

New challenges arise for complex boundaries in multiple dimensions, and a different strategy is needed. In the finite-difference time-domain (FDTD) method in computational electromagnetics $[13,14]$, one approach is to employ staircasing, i.e., replacing the actual boundary with a nearby boundary following grid faces (for discussion see, e.g., Zhao and Wei [15]), though this is not ideal. In a 2D problem with a curved boundary, we define similar staircased contours on either side that approach but do not cross the actual boundary. These mark the edges of the support to either side and allow exact moments to be calculated for use in projection and reconstruction. The reconstruction takes place at the boundary by appropriate 
shifts in the 2D moments, equivalent to mild polynomial extrapolation. (A related but different polynomial extrapolation of wavelet series has been used to handle edge effects in finite wavelet bases before [16].) As the scale is reduced, the average distances between the actual and staircased contours decreases and high-order reduction in error is achieved. There is some nonuniformity in the convergence of pointwise errors, but this is not fatal. In this way, 2D Gibbs oscillations are avoided, and confidence is gained that extension to arbitrary boundaries and higher dimensions will be effective.

The paper is organized as follows. In Section 2 the basics are discussed for wavelet projection and reconstruction via convolutional filters depending on moments. In Section 3 the generalization to include boundaries in 1D is made, and the further generalizations to include boundaries in 2D are made in Section 4. A brief summary of conclusions is given in Section 5, followed by appendices.

\section{Wavelet projection and reconstruction filters}

\subsection{Wavelet transforms}

Orthogonal compact-support Daubechies-type wavelet families [1,17] are characterized by $\phi(x)$ and the mother wavelet (or wavelet function) $\psi(x)$. These are limited in support to the interval $0 \leq x \leq L-1$, where $L$ is an even integer. Each may be expressed as a finite sum of squeezed and translated copies of the scaling function, 


$$
\phi(x)=\sum_{k=0}^{L-1} c_{k} \phi(2 x-k), \quad \psi(x)=\sum_{k=0}^{L-1} d_{k} \phi(2 x-k),
$$

where $c_{k}$ and $d_{k}$ are known two-scale coefficients that are different for each wavelet family. More generally, it is useful to define orthonormal functions formed via scaling by $\lambda$ and translating in units of $\lambda$,

$$
\phi_{k}^{\lambda}(x)=\lambda^{-1 / 2} \phi(x / \lambda-k), \quad \psi_{k}^{\lambda}(x)=\lambda^{-1 / 2} \psi(x / \lambda-k) .
$$

A general function may then be expanded as

$$
\begin{aligned}
& f(x)=\sum_{k} f_{k}^{\lambda} \phi_{k}^{\lambda}(x) d x+O\left(\lambda^{M}\right), \\
& f_{k}^{\lambda}=\int f(x) \phi_{k}^{\lambda}(x) d x,
\end{aligned}
$$

where $M$ is the approximation order ( $L / 2$ for Daubechies wavelets). The error terms represent contributions from wavelet-function subspaces at scales $\lambda$ and finer by powers of 2 . An equivalent multiresolution expansion may be generated by repeated use of the two-scale Eqs. (2) to replace scaling functions on one scale by scaling functions and wavelet functions on the next coarser scale.

If $f(x)$ is a polynomial of degree $<M$, Eq. (3) is exact with no error term since the wavelet functions are constructed to be orthogonal to $1, x, \ldots, x^{M-1}$ and the coefficients can be exactly 
calculated. For more general smooth functions, the $f_{k}^{\lambda}$ are calculated numerically and assumed to have errors $O\left(\lambda^{M}\right)$ or better for Eq. (3) to hold. The latter stipulation can be assured using numerical quadrature of order $r \geq M$ for the projection integral of Eq. (4) [3, 4],

$$
f_{k}^{\lambda}=\lambda^{1 / 2}\left\lfloor\sum_{q=0}^{r-1} \omega_{q} f\left(k \lambda+x_{q} \lambda\right)+O\left(\lambda^{r}\right)\right\rfloor,
$$

where the weights $\omega_{q}$ and the quadrature nodes $x_{q}$ are usually chosen not to depend on $k$ or $\lambda$. A simple choice for order $r=L$ is $x_{q}=q$ for $0 \leq q \leq L-1$, corresponding to uniform spacing of the $L$ quadrature points across the support of $\phi_{k}^{\lambda}$. Practically, this quadrature strategy allows $r$ to exceed $L$ somewhat, utilizing samples of $f(x)$ slightly outside the support of the scaling function to further reduce the error in the approximate projection integral. For large enough $r$, the quadrature will inevitably diverge, limiting absolute accuracy. The projection error is then further reduced by decreasing $\lambda$ while maintaining moderately high values of $r$, capturing dynamic variations of $f(x)$ on finer scales. Tuning of both parameters $r$ and $\lambda$ in concert is an effective way to control projection error as tightly as necessary without wildly oversampling $f(x)$. The projection integration in Eq. (5) is alternatively regarded as a forward discrete wavelet transform step that maps discrete real-space samples to projection integrals with an $O\left(\lambda^{r+1 / 2}\right)$ error. The multiplicative factor $\lambda^{1 / 2}$ is due to the normalization of the basis functions.

The corresponding inverse discrete wavelet transform or reconstruction step can be obtained from the wavelet series expansion in Eq. (3) by sampling $x$ at integer multiples of $\lambda$. The error involved is nominally reconstruction error, but, as noted above, the presence of the 
$f_{k}^{\lambda}$ means that their $O\left(\lambda^{r}\right)$ errors will dominate if they are calculated with quadrature order $r<$ $M$. If $r \geq M$ in projection, the formal leading reconstruction error is $O\left(\lambda^{M}\right)$, but there are still projection error contributions at higher orders. These decrease in importance as $\lambda$ is decreased, but for finite $\lambda$ we only consider reconstruction error as "pure" when the $f_{k}^{\lambda}$ are numerically exact. The $O\left(\lambda^{M}\right)$ limiting behavior for reconstruction errors occurs since $x^{p}$ for $p<M$ is orthogonal to all wavelet functions; all the lower powers are strictly in the scaling function subspace. In spite of this apparent best-case $O\left(\lambda^{M}\right)$ scenario, there have been successful efforts to improve the reconstruction error order based solely on (assumed highly accurate) values of the $f_{k}^{\lambda}$. Keinert and Kwon [5] introduced an auxiliary series with the same coefficients but with transformed functions that accomplished this goal. Neelov and Goedecker [6] derived convolution coefficients for $n \leq L$ neighboring values $f_{k}^{\lambda}$ that would directly provide higherorder $O\left(\lambda^{n}\right)$ pointwise estimates of the original function. As they discuss, their filter elements for order $L$ are the same as the quadrature coefficients $\omega_{q}$ for $r=L$ and $x_{q}=q$ with $q=0,1, \ldots, L-1$. Thus the same filter can be used for projection and reconstruction with error order up to twice the approximation order. These results give the reconstruction error more tunability and inspire a search for extensions.

\subsection{Higher-order reconstruction and differentiation filters}

We now build a generalized theory for reconstruction filters accurate beyond the approximation order and even beyond the length $L$. These include Daubechies and leastasymmetric or symlet families with $M=L / 2$ [1], as well as Coiflet or generalized Coiflet functions with $M<L / 2$ [7]. Consider the scaling function moments 


$$
m_{p}=\int x^{p} \phi(x) d x
$$

which are straightforwardly calculated by recursion [18]. These enable calculation of the shifted monomial projection integrals

$$
\begin{aligned}
x_{p k \tau} & =\int(x-\tau)^{p} \phi(x) d x \\
& =\sum_{p^{\prime}=0}^{p}\left(\begin{array}{l}
p \\
p^{\prime}
\end{array}\right)(k-\tau)^{p-p^{\prime}} m_{p^{\prime}},
\end{aligned}
$$

using changes of variables and the binomial expansion. The parameter $\tau$ is a simple shift that can be chosen freely.

Let us form these quantities into the square $n \times n$ matrix $\mathbf{X}_{\kappa \tau}$, where the rows are labeled by $p=0,1, \ldots, n-1$ and the columns by $q=0,1, \ldots, n-1$, with $k=\kappa+q$ and $\kappa$ being the starting value of $k$,

$$
\left(\mathbf{X}_{\kappa \tau}\right)_{p q}=x_{p, \kappa+q, \tau}
$$

Thus, this is a matrix of moments distributed over a series of $k$ values. (It turns out that the transpose of the $\tau=0$ version of the matrix is used in the paper by Keinert and Kwon [5].) We 
are interested, for a general function $f(x)$, in convolution of its projections with the inverse of this matrix. Using Eq. (4), Taylor expansion of $f(x)$ around $\tau \lambda$, and a change of integration variable, we find

$$
\begin{aligned}
\sum_{q=0}^{n-1} f_{\kappa+q}^{\lambda}\left(\mathbf{X}_{\kappa \tau}^{-1}\right)_{q p^{\prime}}=\sum_{q=0}^{n-1} \int f(x) \phi_{\kappa+q}^{\lambda}(x) d x\left(\mathbf{X}_{\kappa \tau}^{-1}\right)_{q p^{\prime}} \\
=\sum_{q=0}^{n-1} \sum_{p=0}^{\infty} \frac{1}{p !} f^{(p)}(\tau \lambda) \int(x-\tau \lambda)^{p} \phi_{\kappa+q}^{\lambda}(x) d x\left(\mathbf{X}_{\kappa \tau}^{-1}\right)_{q p^{\prime}} \\
=\sum_{p=0}^{n-1} \frac{1}{p !} f^{(p)}(\tau \lambda) \lambda^{p+1 / 2} \sum_{q=0}^{n-1} x_{p, \kappa+q, \tau}\left(\mathbf{X}_{\kappa \tau}^{-1}\right)_{q p^{\prime}}+O\left(\lambda^{n+1 / 2}\right) \\
=\frac{1}{p^{\prime} !} f^{\left(p^{\prime}\right)}(\tau \lambda) \lambda^{p^{\prime}+1 / 2}+O\left(\lambda^{n+1 / 2}\right) .
\end{aligned}
$$

Rearranging yields the result

$$
f^{(p)}(\tau \lambda)=p ! \lambda^{-p-1 / 2} \sum_{q=0}^{n-1} f_{\kappa+q}^{\lambda}\left(\mathbf{X}_{\kappa \tau}^{-1}\right)_{q p^{\prime}}+O\left(\lambda^{n-p}\right)
$$

There are several things to note. (1) The $p=0$ column of $\mathbf{X}_{\kappa, \tau}^{-1}$, when convolved with coefficients $f_{\kappa+q}^{\lambda}$, produces samples of $f(x)$ with error $O\left(\lambda^{n}\right)$; similarly, the $p=1$ column produces samples of $d f / d x$ with error $O\left(\lambda^{n-1}\right)$, and so on. (2) Since $n$ has not been restricted so far, it can exceed the 
approximation order. In practice, $n$ and the starting index $\kappa$ can both be varied so as to optimize the accuracy of the reconstructed samples. (3) If $\tau$ and $\kappa$ are both changed by some integer $i$, it can be seen from Eqs. (7) and (8) that $\mathbf{X}_{\kappa+i, \tau+i}=\mathbf{X}_{\kappa, \tau}=\mathbf{X}_{0, \tau-\kappa}$ and there is only one $\mathbf{X}$ matrix to be calculated. However, $\tau$ takes non-integer values while $\kappa$ does not. Thus one can interpolate the reconstruction of the function or its derivatives continuously by use of $\tau$. For efficient variation of $\tau$ over a range, it is also possible to perform the inversion only for $\tau=0$ (or some other value of $\tau$ chosen, e.g., to reduce the condition number of the matrix) and to relate this to results in the target range of $\tau$ through simple linear transformations.

\subsection{Symbolic form for inverse matrix}

As just suggested, the numerical inversion of $\mathbf{X}_{\kappa, \tau}$ can become more difficult at high $n$ due to poor condition numbers. It is therefore useful that there are symbolic forms for the elements of the inverse. In the case $n=L$ that Neelov and Goedecker discuss [6], they show that the Daubechies reconstruction filter is the same as the quadrature projection filter, which is known in terms of Lagrange interpolating polynomials and scaling function moments [3]. Investigating other cases and other columns of $\mathbf{X}_{\kappa, \tau}^{-1}$, it has been possible to obtain explicit forms for all columns (derivative orders) and for general filter lengths $n$. We simply quote the result. The Lagrange polynomials relative to position $\kappa$ are

$$
L_{n q}(x-\kappa)=\prod_{\substack{q^{\prime}=0 \\ q^{\prime} \neq q}}^{n-1} \frac{x-\kappa-q^{\prime}}{q-q^{\prime}} .
$$


The $\mathbf{X}_{\kappa \tau}^{-1}$ elements involve derivatives of these polynomials around $x=\tau$,

$$
\left(\mathbf{X}_{\kappa \tau}^{-1}\right)_{q p}=\sum_{p^{\prime}=0}^{n-1-p}(-1)^{p^{\prime}}\left(\begin{array}{c}
p+p^{\prime} \\
p
\end{array}\right) a_{p^{\prime}} \frac{1}{\left(p+p^{\prime}\right) !} L_{n q}^{\left(p+p^{\prime}\right)}(\tau-\kappa),
$$

where the coefficients $a_{p^{\prime}}$ satisfy

$$
\sum_{p^{\prime}=0}^{p}(-1)^{p^{\prime}}\left(\begin{array}{l}
p \\
p^{\prime}
\end{array}\right) a_{p^{\prime}} m_{p-p^{\prime}}=\delta_{p 0}
$$

These coefficients may be solved by considering one value of $p$ at a time. For example, using $m_{0}$ $=1$, the equation for $p=0$ gives $a_{0}=1$, that with $p=1$ gives $a_{1}=m_{1}$, etc. This pattern continues up to $a_{L-1}=m_{L-1}$ as a consequence of known relations for the moments such as $m_{2}=m_{1}^{2}$, $m_{4}=4 m_{1} m_{3}-3 m_{1}^{4}$, etc, that hold for $p<L[3]$ (see also [6]). For $p \geq L$, one can still calculate $a_{p}$ and $m_{p}$, but they are no longer equal. The results for $p=0$ (reconstruction) were quoted and used already in solely filter-based quantum eigenvalue problems by Acevedo, et al. [7]. The practical limitation for $n$ is that very large powers $p$ cause loss of precision in the recursion. To some extent this can be compensated in intermediate calculations by switching to moments expanded around the first moment. Nevertheless, just as with the order $r$ in projection, the order $n$ used in reconstruction can only be increased so far. When reconstruction errors start to diverge with $n$, greater accuracy is improved only by reducing the scale.

\subsection{Example}


Here we examine pure reconstruction error without interference from projection error. Reconstruction/differentiation filters are applied to numerically exact projections of the function $f(x)=H_{4}(x) \exp \left(-x^{2} / 2\right)$ with the Hermite polynomial $H_{4}$, which represents an excited wavefunction in vibrational quantum mechanics. The exact function and its first two derivatives are shown in the top row of Fig. 1, each scaled to maximum absolute value 1 for purposes of ready comparison. Calculations are carried out within Mathematica using 20 significant digits of precision. The short $L=6$ Daubechies scaling functions $\phi_{k}^{\lambda_{j}}(x)$ are used, where $\lambda_{j}=\lambda_{0} / 2^{j}$, with $\lambda_{0}=1 / 2$ and $j=0,1, \ldots, 7$. Fully converged coefficients $f_{k}^{\lambda}$ are calculated using $r=10$ numerical quadrature at level $j=7$ and recursion by the two-scale relations in Eqs. (1) to smaller $j$ (coarser scales). The errors in the second and third rows correspond to calculations using the first three ( $p$ $=0,1$ and 2) columns of $\mathbf{X}_{\kappa \tau}^{-1}$. The errors oscillate about the true values, the downward spikes representing oscillation through 0 . The spatial periods of the error are set by the dynamic variation of the functions (insensitive to $j$, and related to well-known correlations of wavelet coefficients across different scales), while the magnitudes systematically decline with increasing $j$. The parameters $\tau$ (relating to output sample location) and $\kappa$ (relating to the starting position of the leftmost scaling function) are fixed relative to each other at a reasonably optimal choice, $v=$ $\tau-\kappa=4$ determined empirically. 

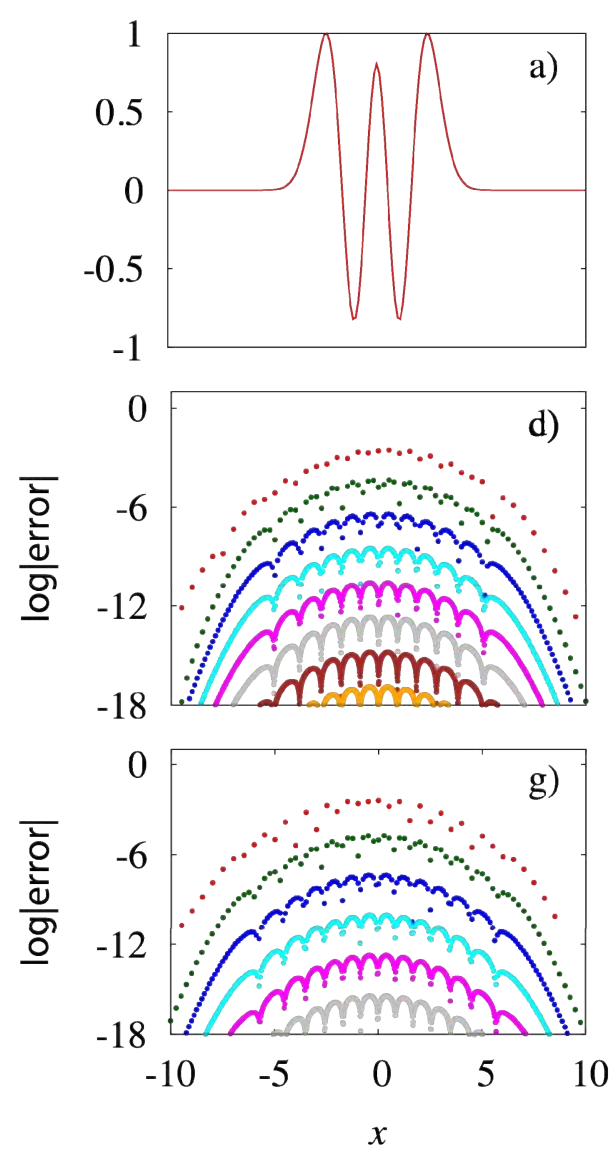
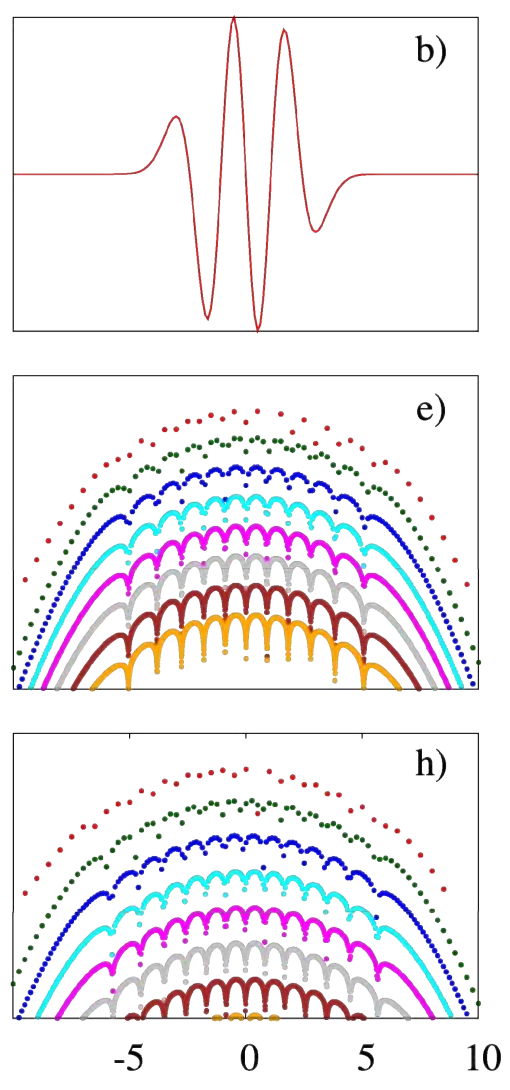
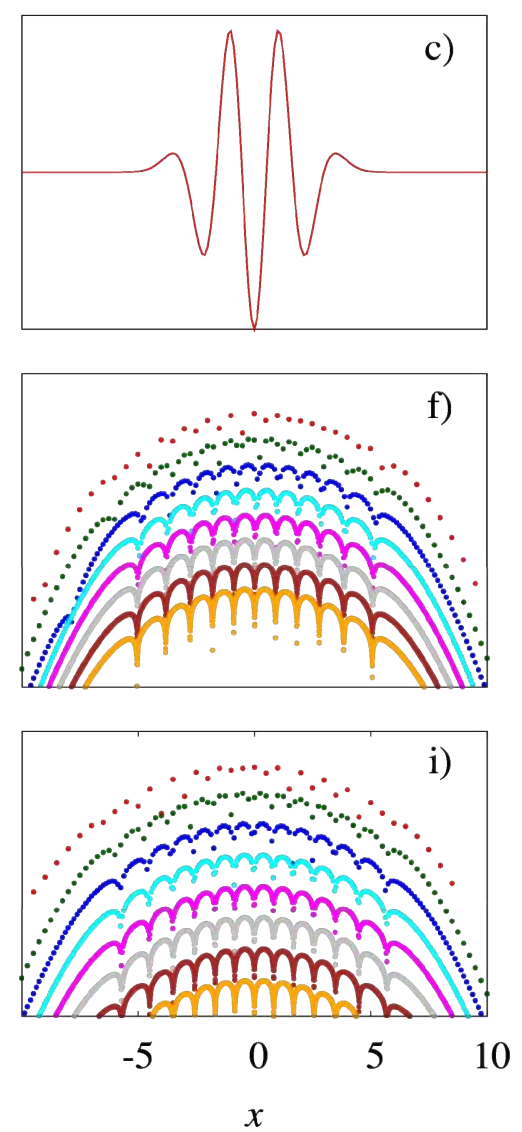

Fig. 1. (a)-(c) Harmonic oscillator wave function with index four and its first and second derivatives, respectively, all scaled to unit maximum value. The bottom two rows show the corresponding base-10 logarithm of absolute errors for $L=6$ Daubechies basis reconstruction/differentiation using filters of lengths (d)-(f) $n=7$ and (g)-(i) $n=9$. Within each of these cases, filters are applied from the appropriate $p=0,1$ and 2 columns of $\mathbf{X}_{\kappa \tau}^{-1}(\tau-\kappa=4)$ to fully converged scaling function coefficients (errors are pure reconstruction errors). Within each plot (d)-(i), the topmost line of errors corresponds to scale $\lambda_{0}=1 / 2(j=0)$, and each successive line lower to scale $\lambda_{j}=\lambda_{0} / 2^{j}(j=1,2, \ldots)$. As expected from Eq. (10), the relative errors are found to scale as $O\left[2^{-j(n-p)}\right]$. 
The second row uses filters of length $n=7$, and the third row uses $n=9$. For comparison, the approximation order of the $L=6$ basis is only $M=3$. In each of the curves in the lower panels, the density of sample points shows the density of basis functions at level $j$. The errors systematically diminish with higher $j$, reflecting dominantly the intrinsic relative scaling behavior $O\left[2^{-j(n-p)}\right]$ as expected from Eq. (10). For a fixed value of $n$, each successive derivative (higher $p$, lower $n-p$ ) in the figures clearly converges more slowly with $j$. At the same time, comparison of the second and third rows shows that this may be compensated by increasing $n$; (d) and (i) are quite similar and have the same value of $n-p$. The bottom line is that global error reduction is much stronger than can be obtained with traditional reconstruction. If the traditional reconstruction is generalized to derivatives, a similar statement is expected to be true.

In Fig. 2 is shown the maximum absolute pure reconstruction error encountered at each value of $j$ for Daubechies bases $L=6,8$ and 10 with $n=2,3, \ldots, 20$. The relative choices of $\tau$ and $\kappa$ used are fixed in each case at $v=L-2$, which is found to be close to optimal for many cases (this can be modified, e.g., if $n$ exceeds $L$ significantly). A steady reduction in error is observed for increases in both $n$ and $j$. Also shown are the results of using the standard reconstruction procedure (black line), which approximately parallels the $n=M=L / 2$ filter behavior for each value of $L$. Similarly the results for using the Neelov and Goedecker $n=L$ filter (blue line) with $v=L-1$, also close to optimal, are approximately parallel in each case to those for the $v=L-2$ and $n=L$ filter used here. 

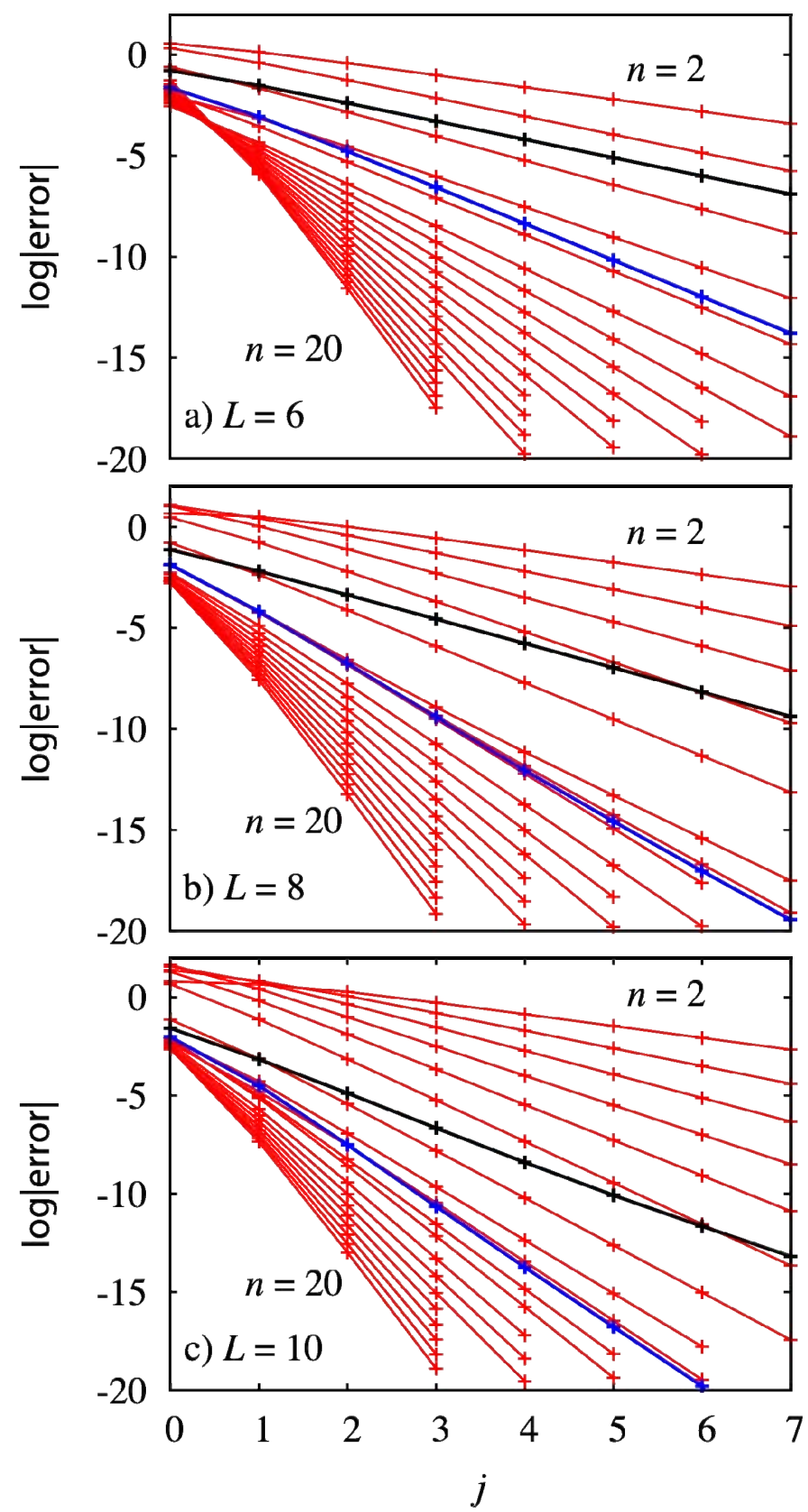

Fig. 2. $\log _{10}$ of absolute maximum pure reconstruction errors for (a) $L=6$, (b) $L=8$ and (c) $L=10$ Daubechies basis with the function in Fig. 1a. The $\lambda_{j}$ values are the same while the range of $n$ has been increased. For comparison, the traditional (black line) and Neelov-Goedecker (blue line) reconstructions have been included. It is clear that the results of using different filter lengths, especially for larger $n$, vary only weakly with $L$. 
The distributed-moment-based reconstruction filters appear to be very effective in extracting high-accuracy reconstructions from high-accuracy coefficients. It is clear from Fig. 2 that the reconstruction errors (devoid of projection errors) depend much more strongly on the filter length $n$ than on the wavelet index $L$. The results for $L=8$ and 10 are very similar while those for $L=6$ are only slightly degraded. A tight accuracy goal is therefore achievable even with relatively short $L$. This may seem surprising, but it is a welcome aspect in many situations, e.g., that described in the next section.

To summarize, we have established a symmetric relationship between the projection and reconstruction steps. The projection integral of a function $f(x)$ for a scaling function on scale $\lambda_{j}$ is calculated as a linear combination of $r$ neighboring samples with an error $O\left(\lambda_{j}^{r}\right)$. Conversely, the reconstruction of $f(x)$ at a particular location is accomplished by taking a linear combination of $n$ neighboring projection integrals. Provided the latter are known at least as accurately, the reconstruction error is $O\left(\lambda_{j}^{n}\right)$. There is some tunability for both $r$ and $n$ for a given $j$, and taking these with high values can be used to make convergence with increasing $j$ rapid so as to avoid excessive numbers of function samples or scaling functions, respectively.

\section{Wavelet projection and reconstruction at boundaries}

\subsection{Tail functions and edge effects}


Use of wavelet bases is more complicated near intrinsic boundaries. For $L \geq 4$, neighboring members of the basis have overlapping supports. For example, the $L=4$ Daubechies scaling functions [1] with $k=0-6$ are shown in Fig. 3 along with their pointwise sum. Theoretically, the sum of all $\phi(x-k)$ is 1 for all $x$. This is verified computationally everywhere except near the edges where contributions from $k=-2,-1,7$ and 8 are missing. Any finite collection of the scaling functions will exhibit such edge effects.

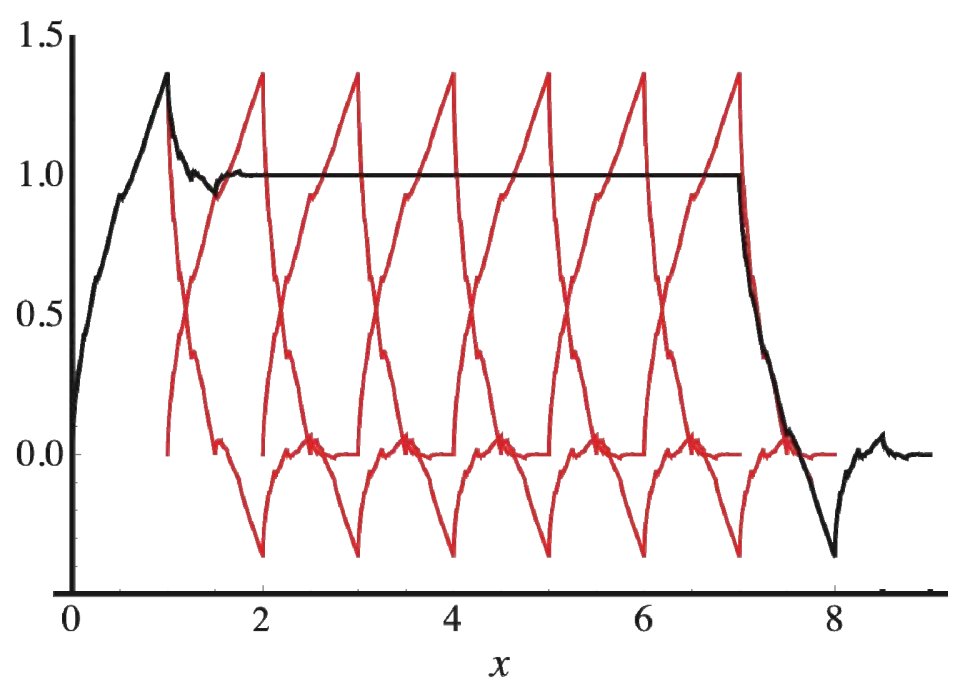

Fig. 3. Individual Daubechies $L=4$ scaling functions $\phi(x-k)$ for $k=0,1, \ldots, 6$ (red curves) and their sum over the combined support (black curve).

For discontinuous functions $f(x)$, description in a finite basis of continuous functions is handicapped by the Gibbs phenomenon, i.e., undershoot/overshoot at the discontinuity. This is most familiar from Fourier analysis but is also present in wavelet analysis $[8,19]$. Unless it is corrected, the error near the discontinuity will completely dominate and prevent any attempt to obtain uniformly high-order error there, and this is a critical issue if one needs to satisfy boundary conditions at the discontinuity. For Fourier pseudospectral solution of Maxwell's 
equations, Min and Grey use post-expansion Gegenbauer reconstruction methods to eliminate the Gibbs phenomenon [20]. Another approach in Fourier problems is the use of one-sided filters [21] to extrapolate to the boundary from one side at a time. This is attractive for use with compact support wavelets since there are only a few basis functions localized near the boundary. An example is shown in Fig. 4, where the $L=6$ Daubechies scaling functions [1] have only 4 members that straddle the position $x=0$, or 6 elements that contact it at all. Modifications to the basis must be made if one-sided filters are to be used while simultaneously avoiding edge effects, but these modifications only need to involve a small number of basis functions.

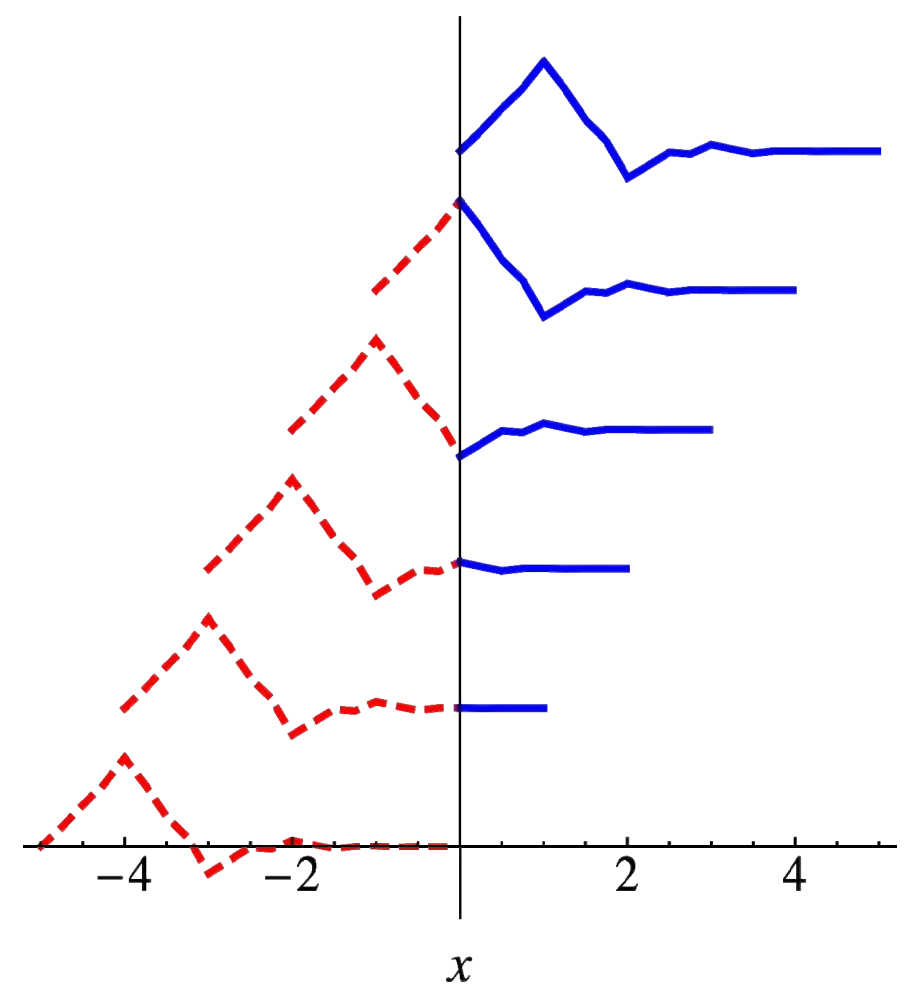

Figure 4. Series of Daubechies $L=6$ scaling functions in neighborhood of 0 , divided into negative and positive $x$ tails, vertically offset for visual clarity. 
Meyer [10] suggested using truncations of the straddling basis functions as part of the basis,

$$
\phi_{k}^{ \pm}(x)=\Theta( \pm x) \phi_{k}(x)
$$

where \pm superscripts denote functions on $x \geq 0$ or $x \leq 0$, respectively, and $\Theta$ is the Heaviside theta function. On either side, the tail functions can be used to maintain approximation order and multiscale decomposition up to $x=0$, though they lose orthonormality between themselves. For Daubechies families, Cohen, et al. [11, 12], answered this problem by re-orthogonalizing $L / 2$ contractions of the tail functions on each side of the boundary. For a finite interval, such modifications at each edge lead to "wavelets on the interval." Such intervalized wavelets have been used in our group for quantum problems with both boundary [22-24] and initial [25, 26] value conditions. However, they do not allow one to beat the approximation order at the edges in reconstruction or, in a related context discussed by Jameson, application of the differentiation matrix [27]. Moreover, the intervalization approach will not generalize well for many multidimensional problems, e.g., solving Maxwell's equations near irregularly-shaped nanoparticles.

Therefore we revert to directly using the non-orthonormal tail functions of Eq. (14). The superscripts \pm are necessary only for $2-L \leq k \leq-1$, but are sometimes extended to neighboring values of $k$ that are not truncated just for notational simplicity. One issue is that the loss of linear independence may lead to large matrix condition numbers and roundoff error in applications [12]. Nevertheless, the $\phi_{k}^{ \pm}(x)$ have been directly useful before, e.g., in wavelet-based calculations for singular-potential scattering [28-30]. Furthermore, it will turn out that there is no 
particular need to use the high- $L$ bases where the ill-conditioning is the most aggravated [12]. This fact is strategically valuable in minimizing the number of basis functions that must be truncated.

Consider the unrestricted expansion of a function $f(x)$ on scale $\lambda$,

$$
f(x) \approx \sum_{k=-\infty}^{\infty} f_{k}^{\lambda} \phi_{k}^{\lambda}(x)
$$

This expansion may be restricted to one side or the other of $x=0$ by multiplication of the appropriate Heaviside function

$$
\begin{aligned}
& \Theta(x) f(x) \approx \sum_{k=2-L}^{\infty} f_{k}^{\lambda} \Theta(x) \phi_{k}^{\lambda}(x)=\sum_{k=2-L}^{\infty} f_{k}^{\lambda} \Theta(x) \phi_{k}^{\lambda+}(x), \\
& \Theta(-x) f(x) \approx \sum_{k=-\infty}^{-1} f_{k}^{\lambda} \Theta(-x) \phi_{k}^{\lambda}(x)=\sum_{k=-\infty}^{-1} f_{k}^{\lambda} \Theta(x) \phi_{k}^{\lambda-}(x) .
\end{aligned}
$$

The expansion coefficients $f_{k}^{\lambda}$ are projection integrals from the unrestricted problem, cf., Eq. (15), and our planned restriction to sample points on one side of $x=0$ will complicate their accurate calculation.

We therefore turn to calculation of truncated or partial projection integrals, 


$$
f_{k}^{\lambda \pm}=\int f(x) \phi_{k}^{\lambda \pm}(x) d x
$$

which may be accomplished exactly for $f(x)$ depending only on powers of $x$ or $x-\tau$. In the case $f(x)=(x-\tau)^{p}$, the quantities

$$
x_{p k \tau}^{ \pm}=\int(x-\tau)^{p} \phi_{k}^{ \pm}(x) d x=\int(x+k-\tau)^{p} \Theta[ \pm(x+k)] \phi(x) d x
$$

are evaluated using truncated moments (sums of unit-interval moments) as discussed in Appendix A. For $k$ values such that there is no truncation, the \pm superscript can be dropped and the integrals are the same as those encountered before. For, say, simple powers of $x$ in a scaled and restricted basis, it is easy to verify that $\int x^{p} \phi_{k}^{\lambda \pm}(x) d x=\lambda^{p+1 / 2} x_{p k 0}^{ \pm}$regardless of the value of $k$, and $\tau \neq 0$ results are only slightly more complicated. Much of the earlier-developed technology for untruncated wavelets extends readily to the truncated case.

While the $f_{k}^{\lambda \pm}$ are not expansion coefficients, their converged sum will be since $\Theta(x)$ $+\Theta(-x)=1$ and therefore $f_{k}^{\lambda+}+f_{k}^{\lambda-}=f_{k}^{\lambda}$.

\subsection{Tail function quadrature integration}

For general functions $f(x)$, numerical wavelet quadrature is modified for use with the tail functions. Assume Lagrange polynomial expansions of $f(x)$ in the neighborhood of $x=0$ using $r$ quadrature points $x_{q}^{ \pm} \lambda$ on the positive or negative side, 


$$
\begin{aligned}
& f(x) \approx \sum_{q=0}^{r-1} f\left(x_{q}^{ \pm} \lambda\right) L_{r q}^{ \pm}(x / \lambda) \\
& L_{r q}^{ \pm}(x)=\prod_{\substack{q \\
q \\
q^{\prime} \neq 0}}^{n-1} \frac{x-x_{q^{\prime}}^{ \pm}}{x_{q}^{ \pm}-x_{q^{\prime}}^{ \pm}}
\end{aligned}
$$

It is possible to exclude the point $x=0$ if a singular behavior occurs there. In any event, one finds for the truncated projection integrals

$$
\begin{aligned}
f_{k}^{\lambda \pm} & =\int f(x) \Theta( \pm x) \phi_{k}^{\lambda}(x) d x \\
& \approx \lambda^{-1 / 2} \sum_{q=0}^{r-1} f\left(x_{q}^{ \pm} \lambda\right) \int L_{r q}^{ \pm}(x / \lambda) \Theta( \pm x) \phi(x / \lambda-k) d x \\
& =\lambda^{1 / 2} \sum_{q=0}^{r-1} \omega_{k q}^{ \pm} f\left(x_{q}^{ \pm} \lambda\right) \\
\omega_{k q}^{ \pm} & =\sum_{p=0}^{r-1} \frac{1}{p !} L_{r q}^{ \pm(p)}(0) X_{p k 0}^{ \pm}
\end{aligned}
$$

The weights $\omega_{k q}^{ \pm}$are independent of $\lambda$ and so may be used for any scale as before in the untruncated problem. Here, however, they depend on the value of $k$ while the samples do not. The quadrature nodes are fixed due to the presence of the boundary while they were allowed to 
"slide" with $k$ in the absence of any boundary (i.e., a different interpolant was used for each $k$ ). Here there are a number of edge filters required, but they may be used for any scale. For the truncated $\phi_{k}^{ \pm}$, some sample points will inevitably fall outside of the support. This is not a problem since samples of the tail functions do not appear explicitly in the formulae. This flexibility has in fact been used for some time with the regular scaling functions, where error is still reduced when taking $r$ somewhat larger than $L$. There are practical limits since, for example, Newton-Cotes quadrature eventually diverges as the order is increased and more distant points are used. When that occurs here, it is taken as a signal to resort to a finer scale.

Refinement of the tail function quadrature requires using the two-scale relations restricted to one side of the boundary. This is described in Appendix B.

\subsection{Tail function higher-order reconstruction}

With the above formulation in place, reconstruction at the edges proceeds exactly as for the regular functions. In analogy to Eq. (8), the square matrices $(\kappa \leq k \leq \kappa+n-1)$

$$
\left(\mathbf{X}_{\kappa, \tau}^{ \pm}\right)_{p, q}=x_{p, \kappa+q, \tau}^{ \pm},
$$

including tail function columns are constructed. For the + case, it is necessary that $\kappa \geq 2-L$ since columns with $k<2-L$ will vanish. Similarly, for the - case, it is necessary that $\kappa+n \leq 0$ to avoid vanishing columns. Other than those restrictions, one may optimize $\kappa$ and $n$ using any 
series of projections even if some are truncated and some are regular. The matrix can then be inverted and used in a proof paralleling Eqs. (9) and (10) to obtain

$$
f^{(p)}(\tau \lambda)=p ! \lambda^{-p-1 / 2} \sum_{q=0}^{n-1} f_{\kappa+q}^{\lambda \pm}\left[\left(\mathbf{X}_{\kappa, \tau}^{ \pm}\right)^{-1}\right]_{q p}+O\left(\lambda^{n-p}\right)
$$

We have not been able to derive a symbolic representation for elements of these inverse matrices as convenient as those for the regular scaling functions. Numerical inversion is thus necessary and poor matrix conditioning must be faced in some cases. This can be partially compensated by scaling, e.g., using $x /(L-2)$, which prevents extremely large values from appearing in $\mathbf{X}_{\kappa, \tau}^{ \pm}$(orthogonal polynomial expansions can also be used for this purpose). There are still small relative values that occur for the shortest tail functions, and their contribution to poor matrix condition cannot be completely removed by scaling. Optimizing the parameters $\kappa$ and $n$ is also useful. Restricting attention to modest $L$, an aspect enabled by higher-order filters, is also advantageous for keeping roundoff error under control. For our exploratory purposes here, we merely use extra precision in construction and inversion of the matrix when needed.

These developments provide a unified higher-order projection-reconstruction formalism including both regular and tail functions. One is not limited to $n \leq M$ (the approximation order), nor even to $n \leq L$, since both quadrature integration and reconstruction are implemented by filters with lengths that may be optimized according to the problem. These filters are designed to work only on one side or the other of a boundary. It remains to examine how reliably the wavelet Gibbs phenomenon is overcome. 


\subsection{Example}

We consider a function which is discontinuous at $x=0$,

$$
f(x)=\operatorname{sgn}(x) \cos (2 x) e^{-|x|} .
$$

This function is expanded in the sets of symlet-8 scaling functions [1] with $\lambda_{j}=2^{-j-1}, j=0,1, \ldots$. Projections converged to more than 16 decimal places are obtained by use of $r=L$ tail function quadrature (Sect. 3.2) and recursion (Appendix B). Errors found are therefore solely those of the reconstruction procedure.

Fig. 5a shows the test function and traditional reconstruction results using exact values of untruncated scaling functions on level $j=0$. For basis functions that straddle the origin, the expansion coefficients are calculated accurately as sums of converged + and - contributions [cf., $\Theta(x)+\Theta(-x)=1]$. The reconstruction errors visible near the discontinuity demonstrate the Gibbs phenomenon. For this standard reconstruction, the absolute errors at the different levels $j=$ $0,1,2, \ldots$ are shown in Fig $5 b$, exhibiting persistent Gibbs phenomenon errors near $x=0$. In any differential equations applications with real-space boundary conditions at the discontinuity, this is the worst possible situation. For comparison, Fig. 5c uses special orthogonal edge (intervalized) scaling functions on each side of $x=0$ with averaging at $x=0$ to avoid Gibbs errors, and Fig. $5 \mathrm{~d}$ uses $n=10$ tail function reconstruction filters both to avoid Gibbs errors and to beat the approximation order as far as rapid error decrease with decrease in scale. Scaling 
function projections in each case are numerically exact so that the comparisons are between pure reconstruction methods. 

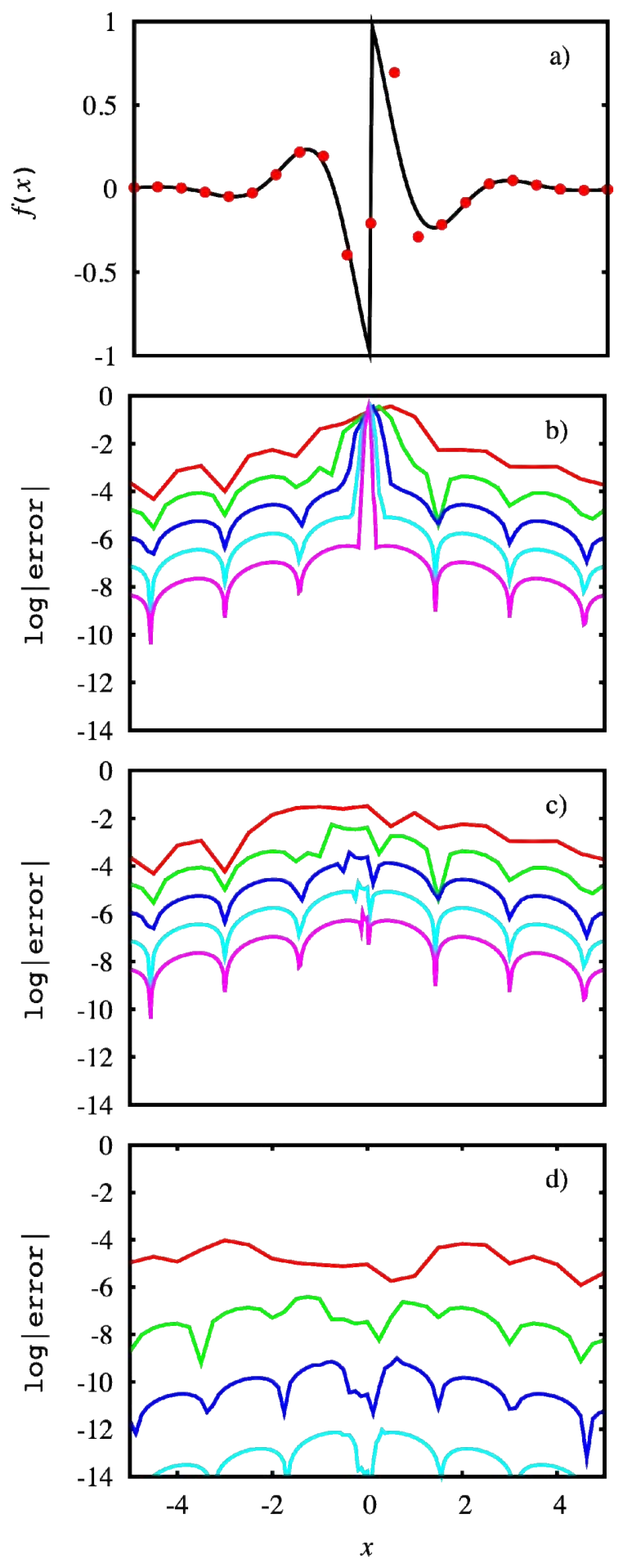

Figure 5. Comparisons of pure reconstruction methods at an interface. (a) Standard reconstruction of the function $f(x)$ of Eq. (26) with a discontinuity at $x=$ 
0 using a symlet- 8 basis of scaling functions on the scale $\lambda=1 / 2$. Gibbs phenomenon errors are visible near the discontinuity. (b) Pointwise $\log _{10}$ absolute errors in standard reconstruction using different scales $\lambda_{j}=2^{-j-1}$ for $j=0,1, \ldots$. Reconstruction errors near $x=0$ narrow with scale but peak error remains the same on each scale. (c) Corresponding errors using orthogonal intervalized scaling functions on each side, avoiding the Gibbs phenomenon but having slow approximation-order-limited overall convergence with scale. (d) Corresponding errors using $n=10$ tail function reconstruction filters, avoiding the Gibbs phenomenon and exhibiting higher order convergence with scale.

For untruncated projections in the symlet- 8 basis, we use filter length $n=10$ and a partially-optimized starting index $\kappa=\tau-6$ to achieve reconstruction at $\lambda_{j} \tau$, requiring calculation of only a single filter. When truncated projections are used, a different filter is generally needed for each value of $\tau$ and the optimal relationship between $\tau$ and $\kappa$ may change. A typical set of choices as used for Fig. $5 \mathrm{~d}$ is shown in Table 1. The errors are indeed found to behave as $2^{-10 j}$, including at the discontinuity. Each doubling in density of basis functions reduces the error by a factor around 1000 . Higher $n$ can be used within limits. If sample points stray too far from the support of the basis function then roundoff errors (using fixed precision) will become more important.

Table 1. Choices of starting indices $\kappa$ for projections/truncated-projections used at each reconstruction index $\tau$. Symlet-8 wavelets are used with filters of length $n$ 
$=10$. Separate reconstructions are performed to the left and right of $x=0$. Far from $x=0$ on either side, the choice $\kappa=\tau+2-L$ is uniformly made.

\begin{tabular}{|ccccc|}
\hline & $x \leq 0$ & & & $x \geq 0$ \\
$\tau$ & $\kappa$ & $\tau$ & $\kappa$ \\
\hline 0 & -10 & 0 & -6 \\
-1 & -10 & 1 & -5 \\
-2 & -10 & 2 & -4 \\
-3 & -10 & 3 & -3 \\
-4 & -10 & 4 & -2 \\
-5 & -11 & 5 & -1 \\
-6 & -12 & 6 & 0 \\
-7 & -13 & 7 & 1 \\
$\ldots$ & $\ldots$ & $\ldots$ & $\ldots$ \\
\hline
\end{tabular}

\section{Extension to 2D}

\subsection{Gibbs oscillations for the fields of a cylindrical nanowire}

A particular application of interest is numerical computation of electromagnetic fields in and around small nanoparticles or nanostructures as dielectric media. For highly symmetric cases such as spheroidal and ellipsoidal nanoparticles, analytical Mie theory and vector harmonics may be used [31,32]. For more realistic nanoparticles, numerical approaches can be taken such as the FDTD [13, 14], finite-element [33], boundary-element [34] and discrete dipole approximation 
[35] methods. Wavelet-based approaches have also been developed, including the multiresolution time domain (MRTD) [36, 37] and wavelet collocation [38, 39] methods.

Nevertheless, there remains a need for Maxwell solvers that have higher-order accuracy, computational practicability, and ability to deal with complex boundaries in multiple dimensions. Even 2D boundaries can be arbitrarily complicated compared to 1D boundary points. Product bases, e.g., $\phi_{k_{x}}^{\lambda}(x) \phi_{k_{y}}^{\lambda}(y)$, will map out rectangular areas of support in 2D space, and these may or may not align with interfaces in real applications. An example of this is light scattering from very rough surfaces in $2 \mathrm{D}$ or $3 \mathrm{D}$. Pan [40] has used a wavelet basis approach that uses the singlepoint sampling properties of the Coiflet basis [1]. However, this is at best limited to approximation-order errors while we want to develop a general wavelet basis description that can overcome both the approximation order and the multidimensional Gibbs phenomena at complex boundaries. This is a tall order.

A prototype application exhibiting these challenges is evaluation of internal and scattered electromagnetic fields at the surface of an infinite conducting cylinder. Cylindrical Ag nanowires, for example, are of current interest for use in transparent conductive films [41]. They were also the subject of investigation by Min, et al. [20], who used 2D Gegenbauer polynomial reconstruction to overcome Gibbs oscillations in transverse-electric (TE) fields calculated using the pseudo-spectral time domain (PSTD) method. This nanosystem is useful as well in our wavelet investigations. Under TE mode excitation, there are only electric field components in the plane transverse to the nanowire axis $z$, i.e., $E_{x}(x, y)$ and $E_{y}(x, y)$, and we may consider this $2 \mathrm{D}$ problem independent of $z$. The electric field components internal to the circular boundary and 
those (incident plus scattered) that are external have exact frequency-domain solutions in the Mie theory description, e.g., by Barber and Hill [31]. At any point on the boundary, the polar-angle component is continuous while the radial component is not. We make the same choice as Min, et al. [20], to use bases in $x$ and $y$ so that there is no particular customization to the curved boundary and the results will provide some indication of what to expect with general boundary shapes.

The electric field components are calculated inside and outside a $25 \mathrm{~nm}$ circular Ag nanowire using $350 \mathrm{~nm}$ excitation. This wavelength is close to both the bulk plasmon peak calculated interpolating the dielectric data of Johnson and Christy [42] and the corresponding $340 \mathrm{~nm}$ surface plasmon peak calculated for the Ag nanowire. The expansion coefficients in the product basis are obtained using $r=3$ 1D numerical quadrature in both $x$ and $y$. This relatively low order limits the number of "contaminated" coefficients in the neighborhood of the discontinuity while allowing the full approximation-order scaling for the $L=6$ basis reconstruction to be achieved in other areas. That is, the projection error matches the approximation order here rather than being reduced to zero.

The top plots of Fig. 6 correspond to two different viewpoints of the real part of $E_{X}$ for excitation with a unit-amplitude incident field. Similarly, the bottom plots correspond to use of ordinary projection and reconstruction. An $L=6$ Daubechies scaling function basis on the scale $\lambda=5 / 2 \mathrm{~nm}$ is used. The "exact" calculations in the top row are evaluated on the same grid, exhibiting some reticulation due to the finite resolution changeover from a square grid to a circular interface and also limited steepness at the discontinuous interface due to limitations of 
plotting software. The wavelet calculations in the bottom row agree everywhere except at the interface, and we identify the strong overshoot/undershoot structure there as dominantly Gibbs oscillations -- it shrinks in width but remains sizeable in magnitude at finer scales as happens in 1D (cf., errors in Fig. 5b). There are also plotting artifacts that increase as the resolution increases, so the resolution chosen is a graphical compromise. The important point is that one cannot use standard reconstruction reliably at the circular boundary using any scale. 

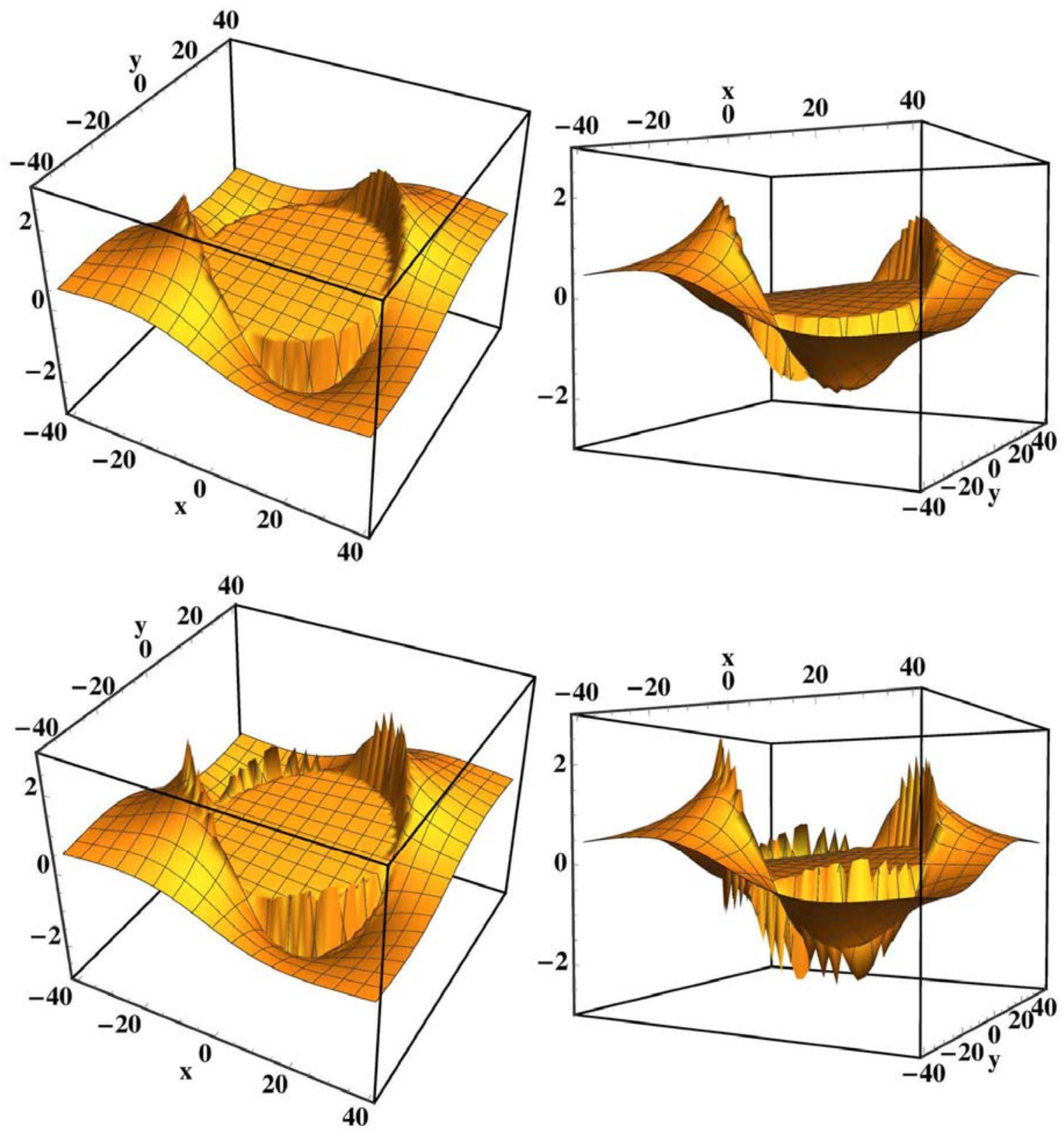

Figure 6. The real parts $\operatorname{Re}\left[E_{X}(x, y)\right]$ of the interior and exterior (scattering) electric fields induced by TE excitation at $350 \mathrm{~nm}$ in a $25 \mathrm{~nm} \mathrm{Ag}$ nanowire All distances are in $\mathrm{nm}$. The top row shows two views of the exact solution on the same grid as the bottom row, which shows the corresponding traditional wavelet reconstruction using $L=6$ Daubechies scaling functions with spacing $\lambda=5 / 2 \mathrm{~nm}$. The behavior of the latter at the 
circular boundary reflects the 2D Gibbs phenomenon as well as is able to be represented by the plotting software.

\subsection{Truncated support and staircased boundaries}

Dividing the plane cleanly into inner and outer regions with truncation at arbitrarily complicated boundaries is problematic since the truncated moments for the $2 \mathrm{D}$ basis functions

$\phi_{k_{x}}^{\lambda}(x) \phi_{k_{y}}^{\lambda}(y)$ will be difficult to calculate accurately. However, a viable strategy is to focus on the $(L-1)^{2}$ sub-squares arising from division of the individual scaling functions into $L-1$ equallength sub-intervals. Multinomial moments for such subsquares can be evaluated easily (see below), thus allowing the 2D generalization of our projection and reconstruction methods used in 1D. The sub-squares can be grouped into inner and outer partial supports, neglecting only those traversed by the circular boundary.

This is indicated in Fig. 7a for the circular nanowire using $\lambda=5 \mathrm{~nm}$ spacing. The inner and outer sub-squares are color-coded, and the contours of closest approach to the circular boundary are highlighted with thicker lines. These staircased contours are reminiscent of those used in FDTD but do not cross the boundary. The corresponding contours for $\lambda=5 / 2$ and $\lambda=5 / 4$ are shown in Figs. 7b and 7c, respectively, though the corresponding sub-squares are suppressed for visual clarity. It is clear that the staircased contours steadily converge to the circle on the average as $\lambda$ decreases, though specific closest-approach distances sometimes happen to remain unchanged from one scale to the next. 

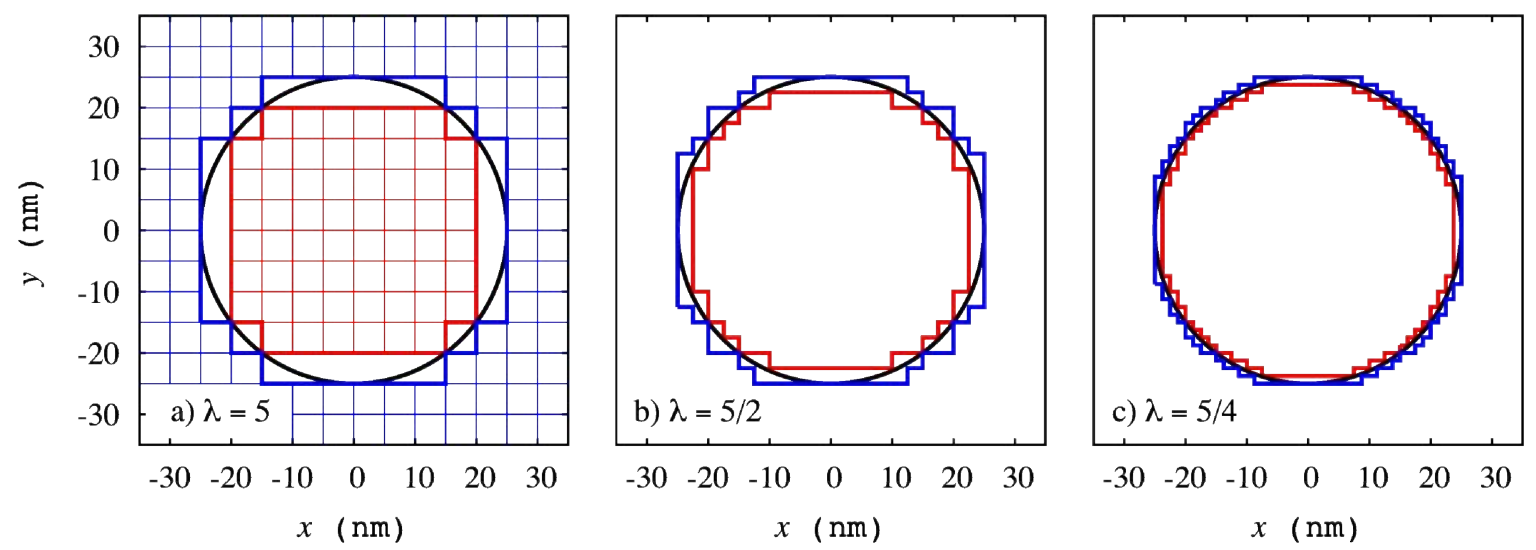

Fig. 7. Circular boundary of radius $25 \mathrm{~nm}$ with inner (thick red) and outer (thick blue) staircased contours of closest approach. Three choices of $\lambda$ are shown: a) $\lambda$ $=5$, b) $\lambda=5 / 2$, c) $\lambda=5 / 4$. For $\lambda=5$, the color-coded purely-inner and purelyouter sub-squares of length $\lambda$ on each side are also shown. As $\lambda$ decreases, the staircased contours approach the circular boundary more closely on the average.

All distances are in $\mathrm{nm}$.

We can express the truncation in terms of window functions. For each value of $\lambda$, let the inner $\Theta^{i, \lambda}(x, y)$ and outer $\Theta^{o, \lambda}(x, y)$ window functions be equal to 1 for a sub-square of area $\lambda^{2}$ entirely on the appropriate side of the boundary ( $i$ or $o$ ) and equal to 0 otherwise. The $2 \mathrm{D}$ basis functions $\Theta^{i / o, \lambda} \phi_{k_{x}}^{\lambda}(x) \phi_{k_{y}}^{\lambda}(y)$ will undergo truncation in some cases by a number of sub-squares. As an example, consider the $L=6$ and $k_{x}=1, k_{y}=1$ case for $\lambda=5$ shown in Fig. 8 . If there were no interface, the support would be the square $\lambda \leq x, y \leq 6 \lambda$. In the presence of the interface, there is an inner support contained in the red staircased curve and an outer support contained in the blue staircased curve. These are the intersections of the basis function's intrinsic support and the two window functions. Thus, there is a $k_{x}=1, k_{y}=1$ truncated basis function in both inner and 
outer bases, but these have disjoint support. The form for truncated moment integrals needed for projection and reconstruction is given in Appendix C.

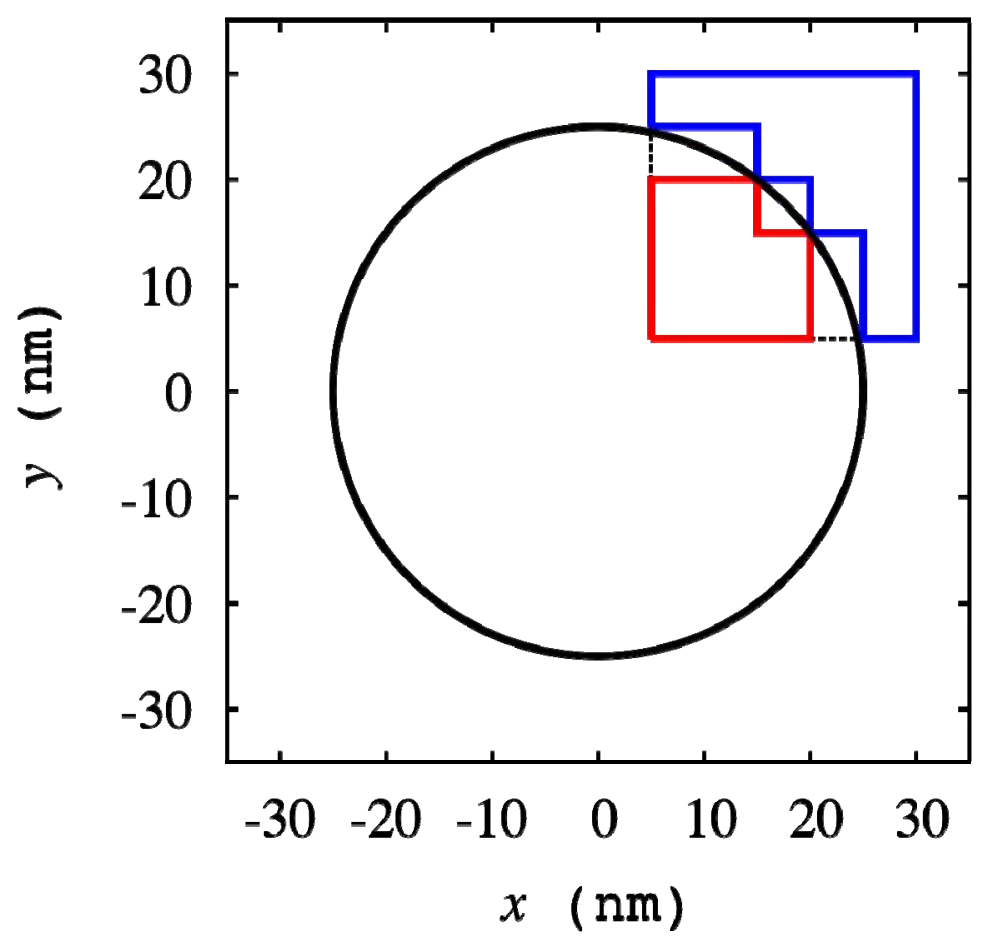

Fig. 8. The full square in the upper right is the support of $\phi_{k_{x}}^{\lambda}(x) \phi_{k_{y}}^{\lambda}(y)$ for $L=6, \lambda=5$, $k_{x}=1$ and $k_{x}=1$. The subregion outlined in red is passed by the window function $\Theta^{i, \lambda}$ of Fig. 7a while the subregion outlined in blue is passed by $\Theta^{o, \lambda}$.

\subsection{Lagrange interpolants in $2 D$}


For truncated integrals over scaling functions and general non-polynomial functions, a quadrature formula can be developed using these moments in conjunction with 2D Lagrangian interpolation based on nodes $\left\{x_{i}, y_{i}\right\}$,

$$
f(x, y) \approx \sum_{i} L_{i}(x, y) f\left(x_{i}, y_{i}\right)
$$

where $L_{i^{\prime}}\left(x_{i}, y_{i}\right)=\delta_{i, i^{\prime}}$. There is a convenient form for these polynomials [43] generalizing that in 1D. In $2 \mathrm{D}$, let the functions $F_{j}\left(x_{i}, y_{i}\right)$ be given by $1, x, y, x^{2}, x y, y^{2}, \ldots$. Then the sample matrix M has elements $M_{i, j}=F_{j}\left(x_{i}, y_{i}\right)$ and the 2D interpolating polynomials are given by

$$
L_{i}(x, y)=\sum_{j} F_{j}(x, y)\left(\mathbf{M}^{-1}\right)_{j, i}
$$

Integrals of $f(x, y) \Theta^{i / o, \lambda}(x, y) \phi_{k_{x}}^{\lambda}(x) \phi_{k_{y}}^{\lambda}(y)$ can then be approximated in terms of function samples and integrals of the type given in Eq. (C.1), i.e., by a $2 \mathrm{D}$ numerical quadrature integration.

\subsection{Choice of $2 D$ nodes and basis functions}

The choice of nodes must still be made, an issue that arises all the time in multivariate integration [44-46]. Our principal goal is to establish whether reconstruction on general boundaries can achieve $O\left(\lambda^{n}\right)$ scaling with tunable $n$. Without any scale refinement, we must 
carry the projection quadrature out to errors of $O\left(\lambda^{r}\right)$ where $r$ is at least as large as $n$. The Lagrange polynomials must therefore contain all multinomial terms in $x$ and $y$ with total order up to $r-1$. There are $r(r+1) / 2$ of these terms and therefore an equal number of quadrature nodes are needed. For simplicity we restrict attention to nodes that are aligned with the $\lambda$-spaced wavelet grid. The nodes are constrained to be in a localized neighborhood, to wit, near a boundary region where reconstruction avoiding the Gibbs phenomenon is to be attempted. The nodes should also be restricted to one side of the appropriate staircased contour. Further constraints are more universal. For example, three points collinear along the $x$ axis will not serve as nodes for the first three functions $1, x$ and $y$. This is reflected in the vanishing of $\operatorname{det}(\mathbf{M})$. Procedurally, we add the functions $F_{j}$ one at a time, search for the nearest not-yet-used node for which $\operatorname{det}(\mathbf{M}) \neq 0$, and then repeat. An example of the nodes chosen for projection is shown in Fig. 9.

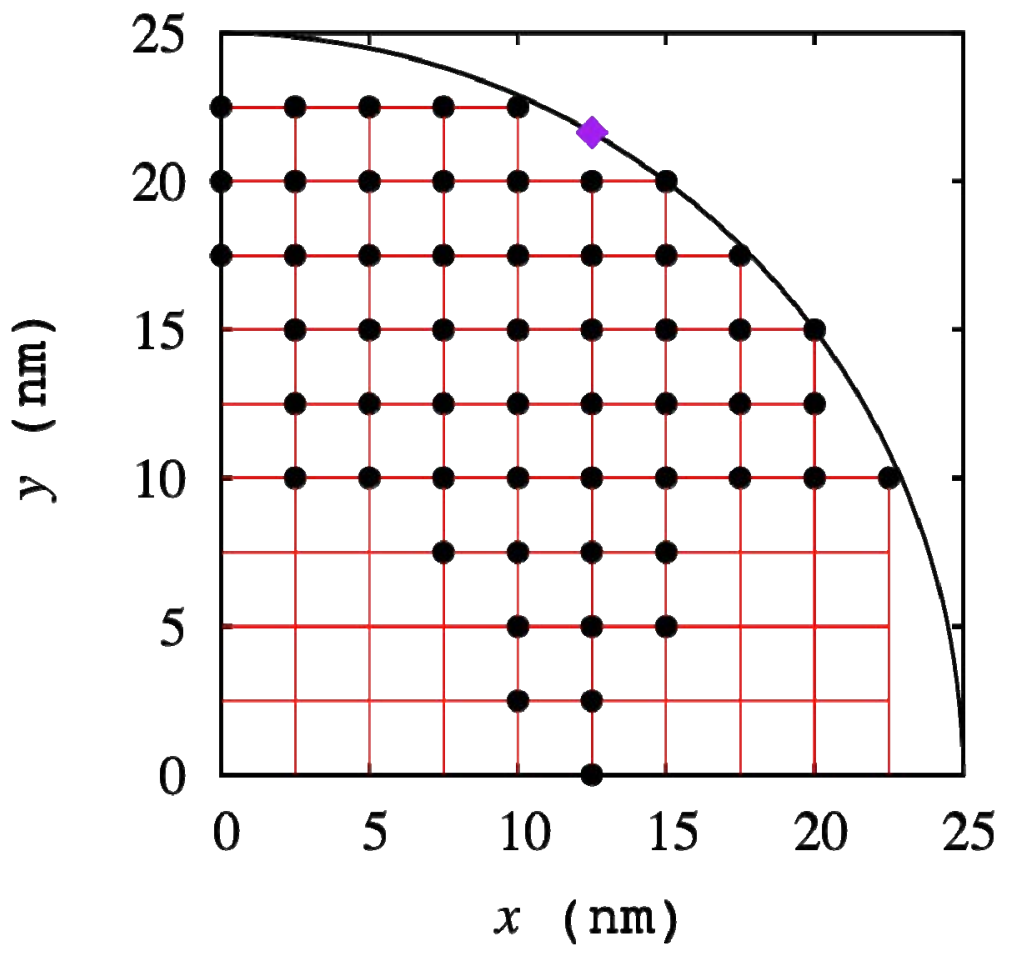


Fig. 9. A typical set of quadrature sample nodes (black dots) chosen for scale $\lambda=5 / 2 \mathrm{~nm}$, order $r=10$, and proximity to the point at $60^{\circ}$ on the circular boundary (purple diamond). The nodes are all inside the boundary but a total span of length $r$ is still swept in both $x$ and $y$.

For calculation of quadrature integration weights as well as formation of the $2 \mathrm{D}$ analog of the distributed moment matrix $\mathbf{X}$ used in reconstruction, it is also necessary to specify a number of $\left(k_{x}, k_{y}\right)$ pairs to form our wavelet basis. These will index the columns of the $\mathbf{X}$ matrix while the rows will be indexed by the set of multinomials $F_{j}(x, y)$ for $j=0,1, \ldots, n(n+1) / 2-1$ needed to ensure that the reconstruction error is $O\left(\lambda^{n}\right)$. There is some choice in the matter though one generally wants to choose basis functions for which the nodes fall in or near their region of support. Among the numerical experiments we have performed, the simplest is to take $n=r$ and to implement a 1:1 match between nodes and $\left(k_{x}, k_{y}\right)$ pairs, e.g., for each node there is a basis function for which $k_{x}$ and $k_{y}$ are simple integer shifts from the nodal coordinates divided by the scale $\lambda$. Moments for quadrature weights in the projection step are calculated from Eq. (C.1) with $\tau_{x}=\tau_{y}=0$. For reconstruction the distributed moments in the $\mathbf{X}$ matrix are calculated with nonzero values of $\tau_{x}$ and $\tau_{y}$, allowing $2 \mathrm{D}$ extrapolation to the nearby boundary.

The unexpected observation in calculations is that the final projection-reconstruction results are independent of the precise choice of $\left(k_{x}, k_{y}\right)$ pairs, though intermediate quantities are not. Comments on this aspect are made later, but for now we just note that different choices are equally satisfactory for this special case $n=r$. 
The choice of $r=10$ is illustrative, but higher $r$ could be used. As it stands, 55 nodal points and basis functions are required to achieve $O\left(\lambda^{10}\right)$ errors. In an untruncated region this represents a savings of 55 of each instead of the 100 that would be used with traditional tensor bases and reconstruction; the latter accommodates higher-scaling terms that are not relevant, e.g., $x^{7} y^{8}$. In 3D with $r=10$, the analogous savings would be 220 instead of 1000 . This is a general consideration for multidimensional calculations when the target is a particular scaling behavior of the error.

\subsection{Example: Reconstruction on the boundary of the Ag nanowire}

In Fig. 10, we examine the accuracy of the extrapolated reconstructions of the internal (plot 10a) and external (plot 10b) $E_{X}$ values at the $60^{\circ}$ point on the circular interface. It is clear that very small errors can be achieved with the combination of finer scales and multi-point projection/reconstruction (no refinement of projection integrals is performed in the following). The variation with increase of $j$ and $r$ is not completely smooth. This is to be expected given the variations in the staircased supports and the choices of nodes. The ideal relative scaling of the error is $O\left(2^{-j} r\right)$. Therefore the ratio $|\operatorname{error}(j, r) / \operatorname{error}(j+1, r)|$ should be $2^{r}$, and $\log _{2}$ of this should be $r$. We plot $1 / r$ times $\log _{2}$ of the observed ratios for $j=2$ and $j=3$ inside errors (plot $10 \mathrm{c}$ ) and the $j=6$ and $j=7$ outside errors (plot 10d). Deviations from unity represent non-ideality of the convergence. The bottom line is that there is at least approximate $O\left(2^{-j} r\right)$ convergence of the error, and the local wavelet methods used here allow control of the error to very tiny levels. 

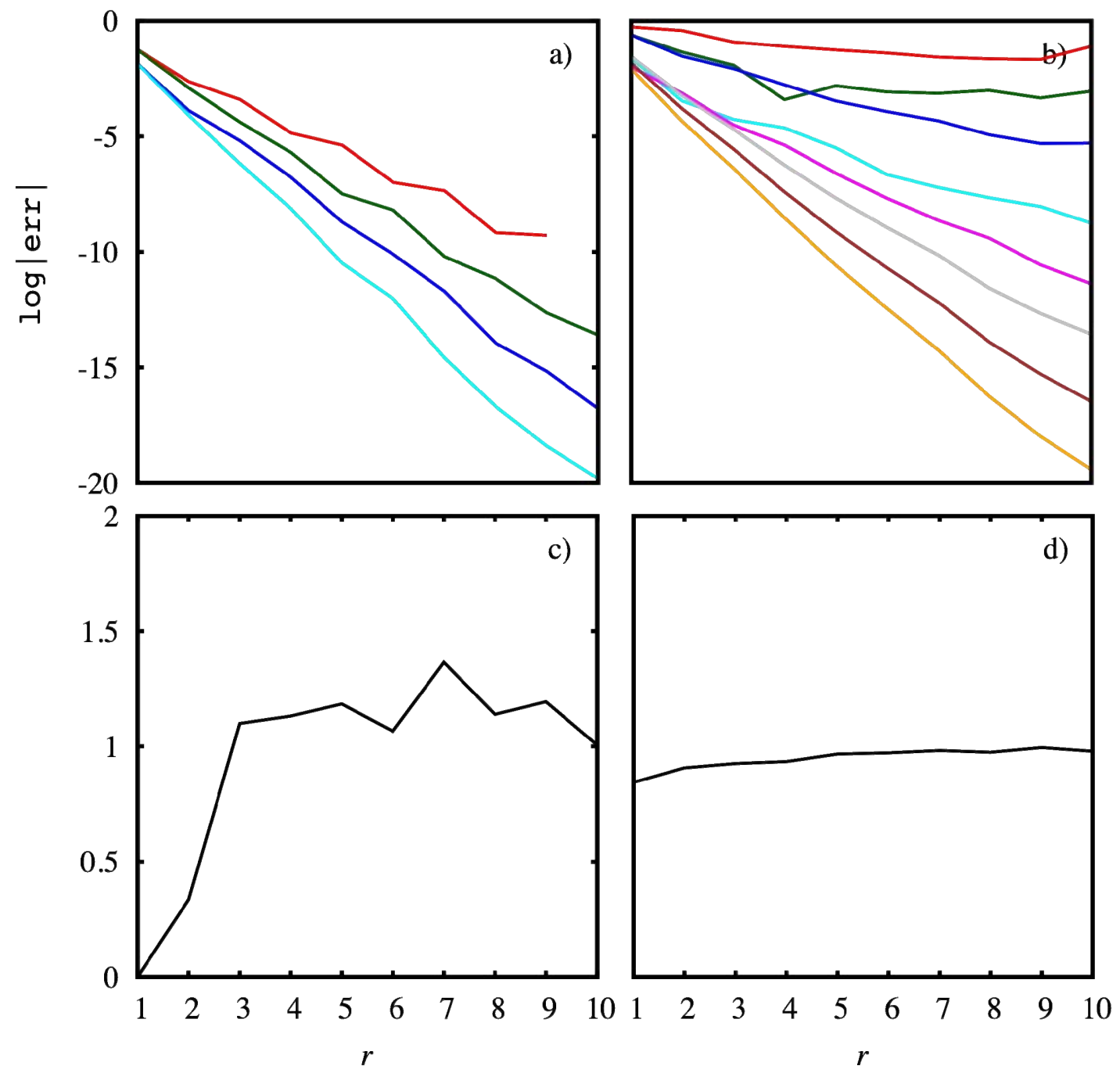

Fig. 10. Plot a) Error in extrapolation of $E_{X}$ from the inner side to the point on the circular boundary at $60^{\circ}$, calculated for $\lambda=5 / 2^{j}, j=0-3$ (descending order). The $j=0$ curve has no solution for choice of quadrature points at $r=10$. Plot b) Same as a), but for the extrapolation of $E_{X}$ from the outer side with $j=0-7$. Divergence is seen for large $r$ with small $j$. Much finer scales are required for extrapolation from outside. The plots c) and d) are corresponding quantities $1 / r$ times $\log _{2}$ of the ratio for the $j=2$ to $j=3$ errors on the 
inside and the $j=6$ to $j=7$ errors on the outside. Differences from unity indicate deviations from the ideal $O\left(2^{-j r}\right)$ error scaling.

There are strong variations in $E_{X}$ and $E_{y}$ outside the interface compared to the relatively placid behavior inside (cf., the real part of $E_{X}$ shown in Fig. 6). From Fig. 10 we see that the outside scale needs to be approximately $2^{4}=16$ times finer to achieve comparably low error. This mismatch is associated with the use of a wavelength near the plasmon resonance of the nanowire, but it is hardly unique that different scales are preferred on different sides of an interface. It is a challenge to take advantage of this for global (e.g., Fourier) bases, but not for finite element methods or those used here, i.e., compact-support wavelets separated/truncated into non-overlapping inner and outer supports. There is no difficulty in the simultaneous use of different scales for different sides of the interface. Thus, at $(\rho, \phi)=\left(25 \mathrm{~nm}, 60^{\circ}\right)$, we mirror the $\lambda$ $=5 / 2 \mathrm{~nm}, r=10$, set of nodes inside the boundary by an analogous $\lambda=5 / 32 \mathrm{~nm}, r=10$, set of nodes (not shown) outside the boundary. Each has its own set of $\left(k_{x}, k_{y}\right)$ basis functions for which projections of $E_{x}$ or $E_{y}$ are calculated. This provides approximately equal reconstruction errors at the target point coming from each side, shown for $E_{X}$ at the point $\phi=60^{\circ}$ in Figs. 11a and $11 b$.

At the same time, the 2D boundary is a curve rather than a point. This makes it of interest that a single set of basis projections (i.e., single set of nodes and single set of basis functions) can be used for different reconstructions that follow the boundary by simple variation of the shifts $\tau_{x}$ and $\tau_{y}$ (wavelet interpolation/extrapolation). Fig. 11a demonstrates a wide swath of angular 
positions for which the error in boundary $E_{X}$ values coming from the inside is well-controlled.

Fig. $11 \mathrm{~b}$ provides the corresponding swath of errors coming from the outside. This has a much narrower region of highest accuracy, of course, consistent with the need for use of a much smaller scale. If it is necessary to cover the whole boundary, nevertheless, this can be done from a finite number of target points at which local basis projections are calculated; these will just need to be more numerous on the outside than the inside of the boundary. Neighboring sets may share nodal points to reduce sampling.
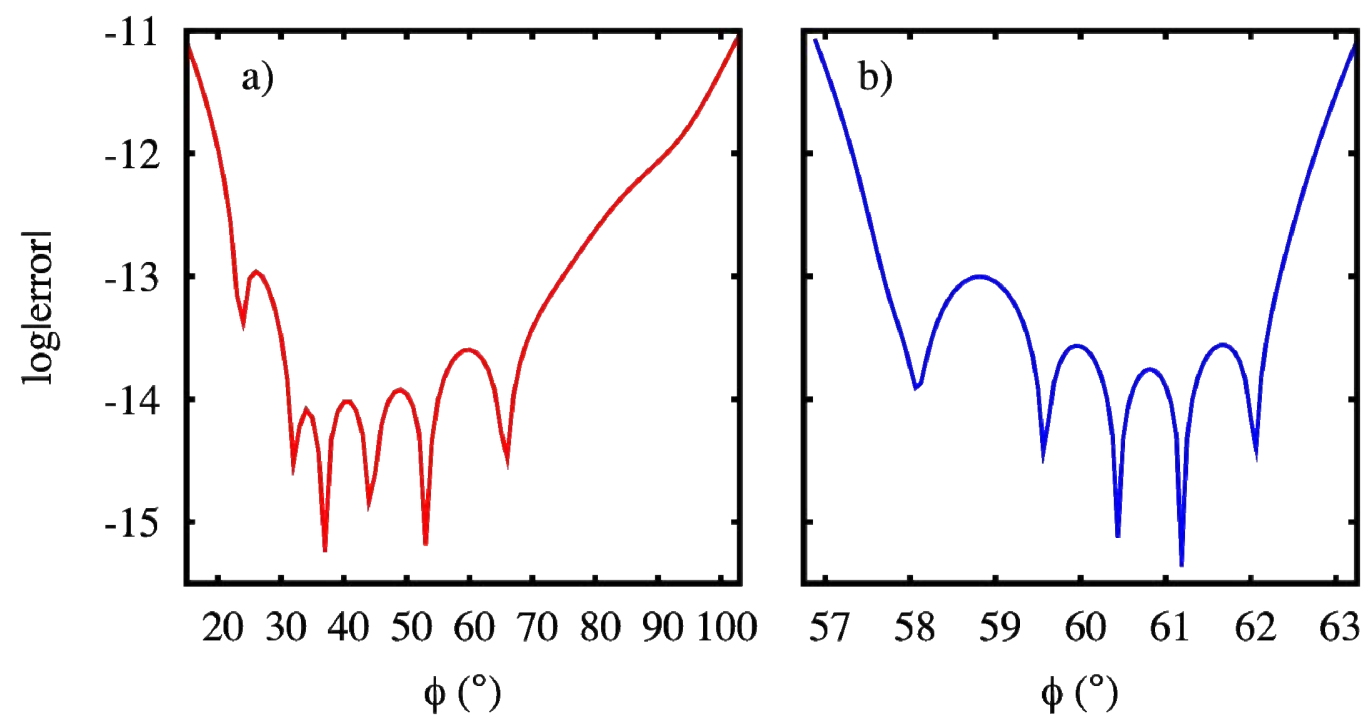

Fig. 11. a) Reconstruction errors in $E_{X}$ along the interface $\rho=R=25 \mathrm{~nm}$ for a range of angle $\phi$ using a single $(r=10, \lambda=5 / 2 \mathrm{~nm})$ projection step based on the interior nodes shown in Fig. 9. b) Corresponding reconstruction errors using a single $(r=10, \lambda=5 / 32)$ set of exterior nodes. Finer scales are needed to achieve comparable error outside the nanowire, reducing the area for which reconstruction can be based on a single set of nodes and set of basis functions. 


\subsection{Perfect reconstruction}

To conclude, we make some comments on how reconstruction results depend, or do not depend, on the specific choice of $\left(k_{x}, k_{y}\right)$ basis functions. If there is no boundary or truncation, "sliding-node" quadrature is ordinarily preferred (Sect. 2.2) where the Lagrange interpolants are translated with $k$. The advantages are that (1) only one set of quadrature weights and one distributed moment matrix need be calculated and (2) the selection of nodes is customized to the support of each basis function. The accuracy then depends on the choice of basis functions, as discussed for the basis parameter $\kappa$ in 1D. Near a boundary the node-sliding must stop for some basis functions since the set of nodes is constrained. In the extreme case, all nodes are chosen to be the same for all included $\left(k_{x}, k_{y}\right)$ basis functions, and then only a single Lagrange interpolant is used. If there is no scale refinement and the choice $r=n$ is made, the final reconstruction (but not the $\mathbf{M}$ and $\mathbf{X}$ matrices) appears to be independent of the basis.

A bit of thought suggested that the unchanging reconstructed values must be those given by the single Lagrange interpolant. This was verified numerically in some simple cases and then verified formally in terms of the $\mathbf{M}$ and $\mathbf{X}$ matrices. There is a philosophical significance to this result since it is a generalization of the "perfect reconstruction" condition usually discussed in terms of simple orthogonal wavelet matrices we would associate with the $r=1$ quadrature limit [1]. The generalization is extensive since it applies to higher-order projection and reconstruction, to one or multiple dimensions, and to truncation (or not) of the basis. However, further exploration of this aspect is deferred for now. 


\section{Conclusions}

In the work described above, we have enhanced the ability of orthogonal compact support wavelets to describe with high-order accuracy and reasonable efficiency functions that are generally smooth but may have discontinuities or derivative discontinuities at specific arbitrarilyshaped interfaces. The latter situation is ubiquitous in EM problems such as describing the near field of a plasmonic nanostructure, but the findings of the paper can be applied more generally.

The basic formalism involves the inversion of an $n$ by $n$ matrix of distributed scaling function moments. The columns of the inverse matrix form convolutional filters that take $n$ neighboring projection integrals for a function $f(x)$ into a particular sample of $f$ (or its derivatives) at a point. This mirrors the use of filters for $r$-point numerical quadrature, which take $r$ neighboring samples of $f$ into a projection integral for a particular scaling function. While these transforms back and forth between real space and wavelet space are not generally perfectly orthogonal, their absolute accuracy can be tightly controlled by using reasonably large $r$ and $n$ to accelerate convergence with decrease in scale $\lambda_{0} / 2^{j}$ so that large $j$ is not needed. That is, if high accuracy is needed in an application, it can be sought in an efficient manner. Implementing the choice of scale locally while maintaining accuracy and efficiency is a direction of future research.

A conclusion drawn is that (pure) reconstruction errors are more strongly dependent on $n$ than on the wavelet length $L$. Wavelet families with higher $L$ have traditionally been used when 
greater approximation order $M$ and accuracy has been needed. In general, longer wavelets require greater computational effort and pose greater awkwardness in dealing with discontinuous boundaries. On the other hand, there may be applications where longer wavelets remain useful, e.g., applications based on projection using single-point quadrature for Coiflets [40] or generalized Coiflets [7] where the error order is at most $M$. For our applications, the arguments align solely in favor of using low- $L$ basis functions. This minimizes the number of straddling functions at a boundary and minimizes the length of the multiscale recursions. Consequently, we have used only short wavelet bases in the calculations of this paper.

The high-order formalism was extended to problems with function discontinuities, where expansions in finite bases of continuous functions always exhibit the Gibbs phenomenon. This was avoided in the wavelet case here by truncating a few basis functions to one side or the other of the interface and calculating truncated distributed moment matrices on either side. Thus, highorder reconstruction beyond the approximation order can be extended straightforwardly to interfaces that align with the wavelet grid. This is impossible to manage in every multidimensional problem one can consider, so a strategy has been developed that can apply to more general interfaces using high-order reconstruction filters that can extrapolate from the wavelet grid to nearby points on the interface. This approach has been applied to projection and reconstruction of the electric field in and around a cylindrical silver nanowire under $\mathrm{CW}$ excitation, which boils down to a $2 \mathrm{D}$ problem with a circular interface. The results indicate that wavelet representations are capable of achieving high-order error in multiple dimensions with complex boundaries. 
While beyond the scope of this paper, the reconstruction filters can be used in the direct solution of differential equations. Neelov and Goedecker have already demonstrated this using their wavelet reconstruction filters in pseudopotential electronic structure calculations [6] and this methodology has been incorporated in the density functional theory package BigDFT [47]. Quite generally, the reconstruction and differentiation filters derived here can all be employed in, for example, wavelet collocation or wavelet Galerkin methods. For boundary value problems, these can be used to control the accuracy in the satisfaction of the boundary conditions (requiring the successful avoidance of the Gibbs phenomenon of course). These may be used in Lagrange multiplier or cost-function-reduction techniques. The reconstruction filter can clearly be of use for Dirichlet conditions. The first derivative filter can similarly be of use for Neumann conditions, or a combination of them for mixed conditions. (Different $n$ for different orders of differentiation $p$ can be used to keep the error order $n-p$ constant.) In any event, it is hoped that the insistence on higher-order accuracy capabilities pursued in the present work will be of use in future methods that are able to shift efficiently between low and high accuracy as needed.

Grateful acknowledgment is made of support in the early stages of this project under the National Science Foundation (NSF) under award CHE-0518476, the NSF and U. S. Department of Defense under NSF award PHY-0755008, and the Robert A. Welch Foundation under award C-1685. Support of later stages of this project was provided under the Independent Research and Development program at NSF while BRJ was a program director there. This paper does not necessarily represent the opinion of NSF in any way.

\section{Appendix A. Unit Interval Moments}


The unit-interval $\phi(x)$ moments

$$
m_{p i}=\int_{i}^{i+1} x^{p} \phi(x) d x
$$

can be calculated by solving a series of linear equations. Using the two-scale relations for the scaling function, one derives the homogeneous equations

$$
m_{p i}=2^{-p-1} \sum_{p^{\prime}=0}^{p}\left(\begin{array}{l}
p \\
p^{\prime}
\end{array}\right) \sum_{k=0}^{L-1} c_{k} k^{p-p^{\prime}}\left(m_{p^{\prime}, 2 i-k}+m_{p^{\prime}, 2 i+1-k}\right)
$$

where the $m_{p i}$ are zero unless $0 \leq i \leq L-2$. The sum of all $L-1$ unit-length moments is then the known regular moment $m_{p}$ defined in Eq. (6), which provides an inhomogeneous equation,

$$
m_{p}=\int_{0}^{L-1} x^{p} \phi(x) d x=\sum_{i=0}^{L-2} m_{p i}
$$

These two equations may be solved together to obtain all of the $m_{p i}$, one value of $p$ at a time. Using changes of variable and the binomial theorem, these lead to the computational formulae for distributed moments

$$
x_{p k \tau}^{+}=\sum_{p^{\prime}=0}^{p}\left(\begin{array}{l}
p \\
p^{\prime}
\end{array}\right)(k-\tau)^{p-p^{\prime}} \sum_{i=\max (0,-k)}^{L-2} m_{p^{\prime} i},
$$




$$
x_{p k \tau}^{-}=\sum_{p^{\prime}=0}^{p}\left(\begin{array}{l}
p \\
p^{\prime}
\end{array}\right)(k-\tau)^{p-p^{\prime}} \sum_{i=0}^{\min (L-2,-1-k)} m_{p^{\prime} i} .
$$

The shift $\tau$ is still a free parameter that may be chosen continuously. The truncated cases, $2-L$

$\leq k \leq-1$, have $k$-dependent summation limits for the innermost sums, while the untruncated integrals depend as before only on the difference $k-\tau$.

\section{Appendix B. Two-Scale Recursions for Tail Function Projection Integrals}

With tail functions included at a boundary, one may use the regular two-scale recursions in the forward direction without reference to any integrals on the "wrong" side of the boundary. This is a minor modification requiring only deletion of some terms from the two-scale convolution in going from finer to coarser scales. Using the first equation in Eqs. (1), we obtain

$$
\begin{aligned}
f_{k}^{\lambda+} & =\int f(x) \Theta(x) \phi_{k}^{\lambda}(x) d x \\
& =\lambda^{-1 / 2} \sum_{k^{\prime}=\max (0,2-L-2 k)}^{L-1} c_{k^{\prime}} \int f(x) \Theta(x) \phi\left(2 x / \lambda-2 k-k^{\prime}\right) d x \\
& =2^{-1 / 2} \sum_{k^{\prime}=\max (0,2-L-2 k)}^{L-1} c_{k^{\prime}} f_{2 k+k^{\prime}}^{\lambda / 2,+},
\end{aligned}
$$




$$
\begin{aligned}
f_{k}^{\lambda-} & =\int f(x) \Theta(-x) \phi_{k}^{\lambda}(x) d x \\
& =\sum_{k^{\prime}=0}^{\min (L-1,-1-2 k)} c_{k^{\prime}} \int f(x) \Theta(-x) \phi\left(2 x / \lambda-2 k-k^{\prime}\right) d x \\
& =2^{-1 / 2} \sum_{k^{\prime}=0}^{\min (L-1,-1-2 k)} c_{k^{\prime}} f_{2 k+k^{\prime}}^{\lambda / 2,-},
\end{aligned}
$$

For those cases where $2 k+k^{\prime}$ is in the untruncated region, the projections without \pm superscripts are implied. High-pass, or wavelet function, convolutions of the partial projections are performed in exactly the same manner except for the use of $d_{k}$ instead of $c_{k}$ coefficients. The inverse wavelet transform step, using both scaling- and wavelet-function coarse projections to obtain scaling-function fine projections, is unchanged in form from the case of untruncated projections except that there are \pm superscripts on the integrals.

\section{Appendix C. Truncated 2D Moment Integrals}

For both numerical quadrature and reconstruction in the 2D basis functions discussed in Section 4.2 , it is necessary to calculate moments truncated by the staircased contours. This is made possible since, for a given product basis function and a given sub-square of area $\lambda^{2}$ in its support, 2D multinomial moments are simply products of the 1D unit-interval moments $m_{p, i}, i=$ $0,1, \ldots, L-2$, described in Appendix A and can be calculated exactly. Independent continuous shifts $\lambda \tau_{x}$ and $\lambda \tau_{y}$ can be accommodated for purposes of reconstruction. The moments for the 
basis function are then sums of contributions from each of the individual subsquares that are not truncated. The 2D integrals needed for both projection and reconstruction are therefore of the general form

$$
\begin{gathered}
\iint \Theta^{i / o, \lambda}(x, y)\left(x-\lambda \tau_{x}\right)^{p_{x}}\left(y-\lambda \tau_{y}\right)^{p_{y}} \phi_{k_{x}}^{\lambda}(x) \phi_{k_{y}}^{\lambda}(y) \\
=\lambda^{p_{x}+p_{y}+1} \sum_{q_{x}=0}^{p_{x}}\left(\begin{array}{l}
p_{x} \\
q_{x}
\end{array}\right)\left(k_{x}-\tau_{x}\right)^{p_{x}-q_{x}} \sum_{q_{y}=0}^{p_{y}}\left(\begin{array}{c}
p_{y} \\
q_{y}
\end{array}\right)\left(k_{y}-\tau_{y}\right)^{p_{y}-q_{y}} \\
\times \sum_{i_{x}, i_{y}} \Theta_{k_{x}+i_{x}, i_{y}+i_{y}}^{i / o,} m_{q_{x}, i_{x}} m_{q_{y}, i_{y}},
\end{gathered}
$$

The last sum over the sub-squares contains a discrete version of the window function denoted as $\Theta_{k_{x}+i_{x}, k_{y}+i_{y}}^{i / o, \lambda}$ that is 1 if the sub-square with indices $\left\{k_{x}+i_{x}, k_{y}+i_{y}\right\}$ is included in the support or 0

if not. If there is no truncation, the last sum is the full product $m_{q_{x}} m_{q_{y}}$ and the entire integral factors. In general truncation prevents overall factorization.

\section{References}

[1] I. Daubechies, Ten Lectures on Wavelets, SIAM Publications, Philadelphia, 1992. 
[2] W. Sweldens, R. Piessens, Quadrature Formulae and Asymptotic Error Expansions for Wavelet Approximations of Smooth Functions, SIAM J. Numer. Anal., 31 (1994) 12401264.

[3] B.R. Johnson, J.P. Modisette, P.J. Nordlander, J.L. Kinsey, Quadrature Integration for Orthogonal Wavelet Systems, J. Chem. Phys., 110 (1999) 8309-8317.

[4] B.R. Johnson, J.L. Kinsey, Quadrature Prefilters for the Discrete Wavelet Transform, IEEE Trans. Signal Proc., 48 (2000) 873-875.

[5] F. Keinert, S.-G. Kwon, High Accuracy Reconstruction from Wavelet Coefficients, Appl. Comp. Harmon. Anal., 4 (1997) 293-316.

[6] A.I. Neelov, S. Goedecker, An efficient numerical quadrature for the calculation of the potential energy of wavefunctions expressed in the Daubechies wavelet basis, J. Comput. Phys., 217 (2006) 312-339.

[7] R. Acevedo, R. Lombardini, B.R. Johnson, Matrix-free application of Hamiltonian operators in Coifman wavelet bases, J. Chem. Phys., 132 (2010) 244112.

[8] H.T. Shim, H. Volkmer, On the Gibbs phenomenon for wavelet expansions, J. of Approx. Theory, 84 (1996) 74-95.

[9] S.E. Kelly, Gibbs phenomenon for wavelets, Appl. Comp. Harmon. Anal., 3 (1996) 72-81.

[10] Y. Meyer, Ondelettes sur l'intervalle, Rev. Mat. Iber., 7 (1992) 115-133.

[11] A. Cohen, I. Daubechies, B. Jawerth, P. Vial, Multiresolution analysis, wavelets and fast algorithms on an interval, C. R. Acad. Sci. Ser. I, 316 (1992) 417-421.

[12] A. Cohen, I. Daubechies, P. Vial, Wavelets on the interval and fast wavelet transforms, Appl. Comp. Harmon. Anal., 1 (1993) 54-81. 
[13] K. Yee, Numerical solution of initial boundary value problems involving Maxwell's equations in isotropic media, IEEE Trans. Anten. Prop., 14 (1966) 302-307.

[14] A. Taflove, S.C. Hagness, Computational Electrodynamics: The Finite-Difference TimeDomain Method, 3rd ed., Artech House, Norwood, MA, 2005.

[15] S. Zhao, G.W. Wei, High-order FDTD methods via derivative matching for Maxwell's equations with material interfaces, J. Comp. Phys., 200 (2004) 60-103.

[16] J.R. Williams, K. Amaratunga, A Discrete Wavelet Transform Without Edge Effects Using Wavelet Extrapolation, J. Fourier Analysis App., 3 (1997) 435-451.

[17] S. Mallat, A Wavelet Tour of Signal Processing, Academic Press, San Diego, 1998.

[18] R.A. Gopinath, C.S. Burris, On the moments of the scaling function $\varphi$, in: Proceedings of the ISCAS-92, San Diego, 1992.

[19] G.G. Walter, X. Shen, Positive estimation with wavelets, Contemporary Mathematics, 216 (1998) 63-79.

[20] M.-S. Min, T.-W. Lee, P.F. Fischer, S.K. Gray, Fourier spectral simulations and Gegenbauer reconstructions for electromagnetic waves in the presence of a metal nanoparticle, J. Comp. Phys., 213 (2006) 730-747.

[21] W. Cai, D. Gottlieb, C.-W. Shu, On One-Sided Filters for Spectral Fourier Approximations of Discontinuous Functions, SIAM J. Num. Anal., 29 (1992).

[22] B.R. Johnson, J.L. Mackey, J.L. Kinsey, Solution of Cartesian and Curvilinear Quantum Equations via Multiwavelets on the Interval, J. Comp. Phys., 168 (2001) 356-383.

[23] A. Maloney, J.L. Kinsey, B.R. Johnson, Wavelets in curvilinear coordinate quantum calculations: $\mathrm{H}_{2}{ }^{+}$electronic states, J. Chem. Phys., 117 (2002) 3548-3557. 
[24] C.D. Griffin, R. Acevedo, D.W. Massey, J.L. Kinsey, B.R. Johnson, Multimode wavelet basis calculations via the molecular self-consistent-field plus configuration-interaction method, J. Chem. Phys., 124 (2006) 134105.

[25] H. Wang, R. Acevedo, H. Mollé, J.L. Mackey, J.L. Kinsey, B.R. Johnson, Multiscale quantum propagation using compact-support wavelets in space and time, J. Chem. Phys., 121 (2004) 7647-7657.

[26] D.K. Sparks, B.R. Johnson, Two-dimensional quantum propagation using wavelets in space and time, J. Chem. Phys., 125 (2006) 114104.

[27] L. Jameson, The Differentiation Matrix for Daubechies-Based Wavelets on an Interval, SIAM J. Sci. Comput., 17 (1996) 498-516.

[28] B.M. Kessler, G.L. Payne, W.N. Polyzou, Scattering Calculations with Wavelets, Few-Body Systems, 33 (2003) 1-26.

[29] B.M. Kessler, G.L. Payne, W.N. Polyzou, Application of wavelets to singular integral scattering equations, Phys. Rev. C, 70 (2004) 034003.

[30] F. Bulut, W.N. Polyzou, Wavelet methods in the relativistic three-body problem, Phys. Rev. C, 73 (2006) 024003.

[31] P.W. Barber, S.C. Hill, Light Scattering by Particles: Computational Methods, World Scientific, Teaneck, N.J., 1990.

[32] C.F. Bohren, D.R. Huffman, Absorption and Scattering of Light by Small Particles, Wiley, New York, 1998.

[33] P. Monk, Finite Element Methods for Maxwell's Equations, Oxford University Press, Oxford Exngland, 2003. 
[34] C. Wieners, J. Xin, Boundary element approximation for Maxwell's eigenvalue problem, Math. Meth. Appl. Sci., 36 (2013) 2524-2539.

[35] V.L.Y. Loke, G.M. Huda, E.U. Donev, V. Schmidt, J.T. Hastings, M.M. Pinar, T. Wriedt, Comparison between discrete dipole approximation and other modelling methods for the plasmonic response of gold nanospheres, Appl. Phys. B, 115 (2014) 237-246.

[36] M. Krumpholz, L.P.B. Katehi, MRTD: new time-domain schemes based on multiresolution analysis, IEEE Trans. Microwave Theory Tech., 44 (1996) 555-571.

[37] M. Zhu, Q. Cao, S. Gao, Studying and Analysis of the Characteristic of the Higher-Order FDTD and MRTD and RK-MRTD Schemes, Appl. Comp. Electromag. Soc., 28 (2013) 380390.

[38] M. Fujii, W.J.R. Hoefer, Interpolating wavelet collocation method of time dependent Maxwell s equations: characterization of electrically large optical waveguide discontinuities, J. Comput. Phys., 186 (2003) 666-689.

[39] H. Li, K.R. Hiremath, A. Rieder, W. Freude, Adaptive Wavelet Collocation Method for Simulation of Time Dependent Maxwell's Equations, in, arXiv, 2012.

[40] G.G.W. Pan, Coifman Wavelets in 3-D Scattering From Very Rough Random Surfaces, IEEE Trans. Anten. Prop., 52 (2004) 3096-3103.

[41] J. Jiu, T. Sugahara, M. Nogi, K. Suganuma, Ag nanowires: large-scale synthesis via a tracesalt-assisted solvothermal process and application in transparent electrodes, J. Nanopart. Res., 15 (2013) 1588:1581-1513.

[42] P.B. Johnson, R.W. Christy, Optical Constants of the Noble Metals, Phys. Rev. B, 6 (1972) 4370-4379. 
[43] K. Saniee, A Simple Expression for Multivariate Lagrange Interpolation, SIAM J. Undergraduate Research Online, 1 (2007).

[44] G.W. Wasilkowski, H. Woźniakowski, Explicit Cost Bounds of Algorithms for Multivariate Tensor Product Problems, J. of Complexity, 11 (1995) 1-56.

[45] M. Gasca, T. Sauer, Polynomial interpolation in several variables, Adv. Comp. Math., 12 (2000) 377-410.

[46] M. Gunzburger, A.L. Teckentrup, Optimal Point Sets for Total Degree Polynomial Interpolation in Moderate Dimensions, in, ArXiv, 2014, pp. 17.

[47] L. Genovese, M. Ospici, T. Deutsch, J.-F. Méhaut, A. Neelov, S. Goedecker, Density functional theory calculation on many-cores hybrid central processing unit-graphic processing unit architectures, J. Chem. Phys. 131 (2009) 034103. 